\title{
Unraveling Tumor-Immune Heterogeneity in Advanced Ovarian Cancer Uncovers Immunogenic Effect of Chemotherapy
}

Alejandro Jiménez-Sánchez ${ }^{*}$, , Paulina Cybulska ${ }^{*}, 2$, Katherine Lavigne ${ }^{*}, 2$, Tyler Walther ${ }^{3}$, Ines Nikolovski ${ }^{4}$, Yousef Mazaheri ${ }^{5}$, Britta Weigelt ${ }^{5}$, Dennis S. Chi ${ }^{2}$, Kay J. Park ${ }^{5}$, Travis Hollmann ${ }^{5}$, Dominique-Laurent Couturier ${ }^{1}$, Alberto Vargas ${ }^{4}$, James D. Brenton ${ }^{1}$, Evis Sala ${ }^{4,6}$, Alexandra Snyder ${ }^{3}$, Martin L. Miller ${ }^{\wedge}, 1$.

* Co-first authors

^ Correspondence: martin.miller@cruk.cam.ac.uk

1. Cancer Research UK Cambridge Institute, University of Cambridge, Li Ka Shing Centre, Robinson Way, Cambridge CB2 ORE, UK.

2. Department of Surgery, Memorial Sloan Kettering Cancer Center, 1275 York Avenue, New York, NY 10065, USA.

3. Department of Medicine, Memorial Sloan Kettering Cancer Center, 1275 York Avenue, New York, NY 10065, USA.

4. Department of Radiology, Memorial Sloan Kettering Cancer Center, 1275 York Avenue, New York, NY 10065, USA.

5. Department of Pathology, Memorial Sloan Kettering Cancer Center, 1275 York Avenue, New York, NY 10065, USA.

6. Department of Radiology, Li Ka Shing Centre, Robinson Way, Cambridge CB2 ORE, UK. 


\section{ABSTRACT}

In metastatic cancer, the role of heterogeneity at the tumor-immune microenvironment, its molecular underpinnings and clinical relevance remain largely unexplored. To understand tumor-immune dynamics at baseline and upon chemotherapy treatment, we performed

5 unbiased pathway and cell type-specific immunogenomics analysis of treatment-naive (38 samples from 8 patients) and paired chemotherapy treated (80 paired samples from 40 patients) high-grade serous ovarian cancer (HGSOC) samples. Whole transcriptome analysis and imagebased quantification of $\mathrm{T}$ cells from treatment-naive tumors revealed ubiquitous variability in immune signaling and distinct immune microenvironments co-existing within the same

10 individuals and within tumor deposits at diagnosis. To systematically explore cell type composition of the tumor microenvironment using bulk mRNA, we derived consensus immune and stromal cell gene signatures by intersecting state-of-the-art deconvolution methods, providing improved accuracy and sensitivity when compared to HGSOC immunostaining and leukocyte methylation data sets. Cell-type deconvolution and pathway analyses revealed that

15 Myc and Wnt signaling associate with immune cell exclusion in untreated HGSOC. To evaluate the effect of chemotherapy on the intrinsic tumor-immune heterogeneity, we compared sitematched and site-unmatched tumors before and after neoadjuvant chemotherapy. Transcriptomic and T-cell receptor sequencing analyses showed that site-matched samples had increased cytotoxic immune activation and oligoclonal expansion of T cells after chemotherapy,

20 which was not seen in site-unmatched samples where heterogeneity could not be accounted for. These results demonstrate that the tumor-immune interface in advanced HGSOC is intrinsically heterogeneous, and thus requires site-specific analysis to reliably unmask the impact of therapy on the tumor-immune microenvironment. 


\section{INTRODUCTION}

It is unclear how the complex interplay between tumor cells and the tumor microenvironment (TME) and their interactions affect treatment outcome in metastatic cancer (Kitamura, Qian, and Pollard 2015; Janssen et al. 2017; Robinson et al. 2017). Investigating this interplay in an advanced disease setting is complicated by the difficulty of obtaining multiple-site tumor samples and the finding that different tumors within the same individual can harbor distinct immune microenvironments (Sridharan et al. 2016; Jiménez-Sánchez et al. 2017a; Reuben et al. 2017; A. W. Zhang et al. 2018). Moreover, interactions between different cell populations of the TME are plastic and can change dependent on extrinsic perturbations such as therapy (Wang et al. 2016). In systems biology, perturbations are often used to infer how individual components of the system are interconnected by analyzing their dynamic behaviour in response to the external stimuli (Aldridge et al. 2006; Geva-Zatorsky et al. 2010; Molinelli et al. 2013). By analogy, immuno-oncology research needs to systematically characterize how oncogenic signaling activity affects the TME and immune infiltration, as well as to explore the variability and plasticity observed across clinical settings such as disease stage, anatomic sites and effect of treatment.

Heterogeneity in cancer spans multiple dimensions: at the molecular level with the presence of genetic intra-tumor heterogeneity (ITH) (Shah et al. 2009; Campbell et al. 2010; Gerlinger et al. 2012), the cellular level with variability observed in infiltration and recruitment of non-tumor cell

45 populations in the TME (Natrajan et al. 2016), and the spatial and population levels where variability is observed both within tumors of the same individual with disseminated disease (Jiménez-Sánchez et al. 2017) and between tumors of different patients (McPherson et al. 2016; Patch et al. 2015). Thus, unbiased and systematic analysis of ITH and TME heterogeneity poses significant challenges. While the study of ITH has been facilitated by computational 
50 approaches to estimate the distribution and co-existence of different tumor clones from largescale somatic mutation data, bioinformatics analysis of TME heterogeneity poses considerable technical difficulties due to the diversity of cell types present in the TME, in particular when distinguishing tumor from non-tumor cell populations based on bulk tumor analysis. Recent genomic approaches to characterize the TME include the development of immune cell

55 deconvolution methods that aim to decompose a mixture of immune cell gene transcripts from bulk expression data (Finotello and Trajanoski 2018). However, no objective side-by-side comparisons of these methods have been implemented (Zheng 2017), and the accuracy and utility of immune cell deconvolution methods for determining TME heterogeneity in clinical settings is uncertain.

60

High grade serous ovarian cancer (HGSOC) is ideally suited to the study of TME heterogeneity owing to its clinical presentation with multisite abdominal disease and standardized treatment with either optimal surgical debulking at diagnosis or delayed primary surgery after neoadjuvant chemotherapy (NACT) (Bowtell et al. 2015). Thus, in HGSOC there is a unique opportunity to study the characteristics of the TME at multiple sites and to observe variation at baseline (diagnosis) and following perturbation with platinum-based chemotherapy, with the underlying hypothesis that the role of the TME may be exposed by its dynamic response to extrinsic perturbations (e.g. chemotherapy). Furthermore, since HGSOC is typically diagnosed when dissemination has already taken place in the peritoneal cavity, this malignancy provides the

70 basis for evaluating the ubiquitousness of intra-patient TME heterogeneity in an advanced, metastatic disease setting. In HGSOC, the low somatic point mutation load, high aneuploidy levels and high copy number alterations have been associated with lack of immunogenicity (Bowtell et al. 2015; A. W. Zhang et al. 2018). Despite the low intrinsic immunogenicity, T cell infiltration plays a major role in predicting HGSOC survival in a primary disease setting (L. 75 Zhang et al. 2003; Ovarian Tumor Tissue Analysis (OTTA) Consortium et al. 2017), and recent 
studies have started to shed light on the interplay between ITH and T cell interactions (A. W. Zhang et al. 2018), as well as the potential effect of chemotherapy on T cell infiltration in HGSOC (Böhm et al. 2016). However, the extent of TME heterogeneity has not been systematically characterized in metastatic disease, including advanced HGSOC, and its underlying mechanisms and role in therapeutic response remain unknown.

To characterize heterogeneity at the level of the TME and to begin to identify the molecular and cellular underpinnings of immune infiltration variability at diagnosis and after perturbation by chemotherapy, we performed a systematic analysis of $>100$ HGSOC samples from treatment-

85 naive and NACT patient cohorts. Our findings confirm that HGSOC is a disease characterized by pervasive TME heterogeneity with distinct immune microenvironments co-existing in different tumor nests within the same individuals at diagnosis. We leverage our rich data sets to create an ensemble computational approach that integrates and improves upon existing immune and stromal cell deconvolution methods, thus enabling us to systematically characterize the TME of HSGOC before and after treatment. We identify oncogenic signaling pathways such as Myc and Wnt that associate with immune cell exclusion when comparing tumors with high cancer cell fraction (high purity) vs low cancer cell fraction (low purity). We find that NACT induces immune activation and specific $\mathrm{T}$ cell clonal expansions in local TMEs, however, intra-patient TME immune heterogeneity can mask such effects. Consequently, systemic immunomodulatory 95 therapies may be ineffective in a subset of tumor sites, thus preventing overall patient benefit. Together, these results show that intra-patient TME heterogeneity is ubiquituos in HGSOC, which could confound clinical outcomes. 
100 RESULTS

\section{Intrapatient Transcriptomic Heterogeneity is Largely Explained by Immune}

\section{Signaling}

To investigate the tumor microenvironment (TME) of HGSOC in a treatment-naive context, we analyzed the transcriptome of 38 primary and metastatic tumor samples from 8 105 treatment-naive patients collected prospectively (Figure 1A, Supplementary Table 1A and B). Primary tumor masses and peritoneal metastases were resected and placed on patient-specific 3D moulds created based on tumor segmentation using high resolution T2-weighted magnetic resonance $(M R)$ images [REF:Weigelt $B$, Vargas $A H$, Selenica $P$, Geyer FC, Mazaheri $Y$, Blecua P, Conlon N, Hoang LN, Jungbluth AA, Snyder A, Ng CKY, Papanastasiou AD, Sosa

110 RE, Soslow RA, Chi DS, Gardner GJ, Shen R, Reis-Filho JS, Sala E. Radiogenomics analysis of intra-tumor heterogeneity in high-grade serous ovarian cancer. BJC (under review)]. Each specimen was placed in the custom-made 3D mould in the operating theatre and was further dissected into sub-specimens according to three multi-parametric imaging-based phenotypically distinct clusters, hereafter referred to as "habitats". Habitats were obtained from MR and 18F-

115 FDG-PET imaging and were defined based on quantitative imaging features that measure water diffusion, micro-capillary perfusion, permeability and metabolic activity (see Methods). We first performed an unbiased clustering analysis of the whole transcriptome. We observed that overall gene expression of tumor samples was highly patient specific, irrespective of anatomical site using t-distributed stochastic neighbor embedding (t-SNE) which accounts for nonlinear relationships (Figure 1B). To focus on well-defined biological processes and signaling pathways, we performed ssGSEA (Hänzelmann, Castelo, and Guinney 2013) using the hallmark gene sets (Arthur Liberzon et al. 2015), stromal and immune gene signatures, and tumor cell fraction 
(purity) using the ESTIMATE algorithm (Yoshihara et al. 2013). We categorized the gene sets into five classes: oncogenic, cellular stress, immune, stromal, and other. Principal component 125 analysis (PCA) showed that most of the gene set expression variation between samples $(60 \%$ of variation) could be explained by oncogenic, immune, and stroma-associated gene sets (Figure 1C, S1A). In contrast to the full transcriptome analysis, the patient specific clustering was less evident, indicating that tumors from different patients share common patterns of pathway activation and non-cancer cell infiltrates. To investigate which gene sets explained 130 most of the observed variance, we computed the principal component feature loadings and displayed them in a variable factor map (Figure 1D). This analysis showed that PC1 (40\% of variation) is largely explained by tumor purity, since immune and stromal vectors had an opposite direction to oncogenic vectors and tumor purity (immune vs oncogenic: $p=3 \mathrm{e}^{-05}$; stromal vs oncogenic: $\left.p=1.3 \mathrm{e}^{-03}\right)$, and PC2 (20\% of variation) further showed a separation of immune, stromal, and cellular stress vectors (immune vs stromal: $p=0.046$; immune vs stress: $p=0.041 ;$ Figure S1B).

Since immune related pathways explained a significant amount of variation between the samples, we further investigated the extent of intra-patient immune heterogeneity by computing 140 the ESTIMATE immune score for each sample. In addition, we included as a reference the immune scores of the samples from a HGSOC case study with $>9$ years of clinical history we previously analyzed (Jiménez-Sánchez et al. 2017) and the immune scores of ovarian cancer samples from The Cancer Genome Atlas (TCGA), which comprises 307 treatment-naive primary tumors (Cancer Genome Atlas Research Network 2011). Overall, the immune scores of 145 our cohort fell within the range expected at the population level (Figure 1E). Some patients (01, 04, 10, and the case study) showed an intra-patient variation comparable to the inter-patient variation observed at the population level by the TCGA ovarian cancer samples, which indicates that within a single individual, complete distinct immune microenvironments can co-exist at 
diagnosis of HGSOC. Also, all patients in the cohort had at least one sample with similar or

150 lower immune score than the progressing and immune excluded tumors of the case study, where distinct tumor-immune microenvironments led to different clinical outcomes (JiménezSánchez et al. 2017). Importantly, consistent with our prior report, we recapitulate the observation that tumors with high immune signaling and immunosuppressive Wnt signaling tend to be mutually exclusive (Figure 1D). 


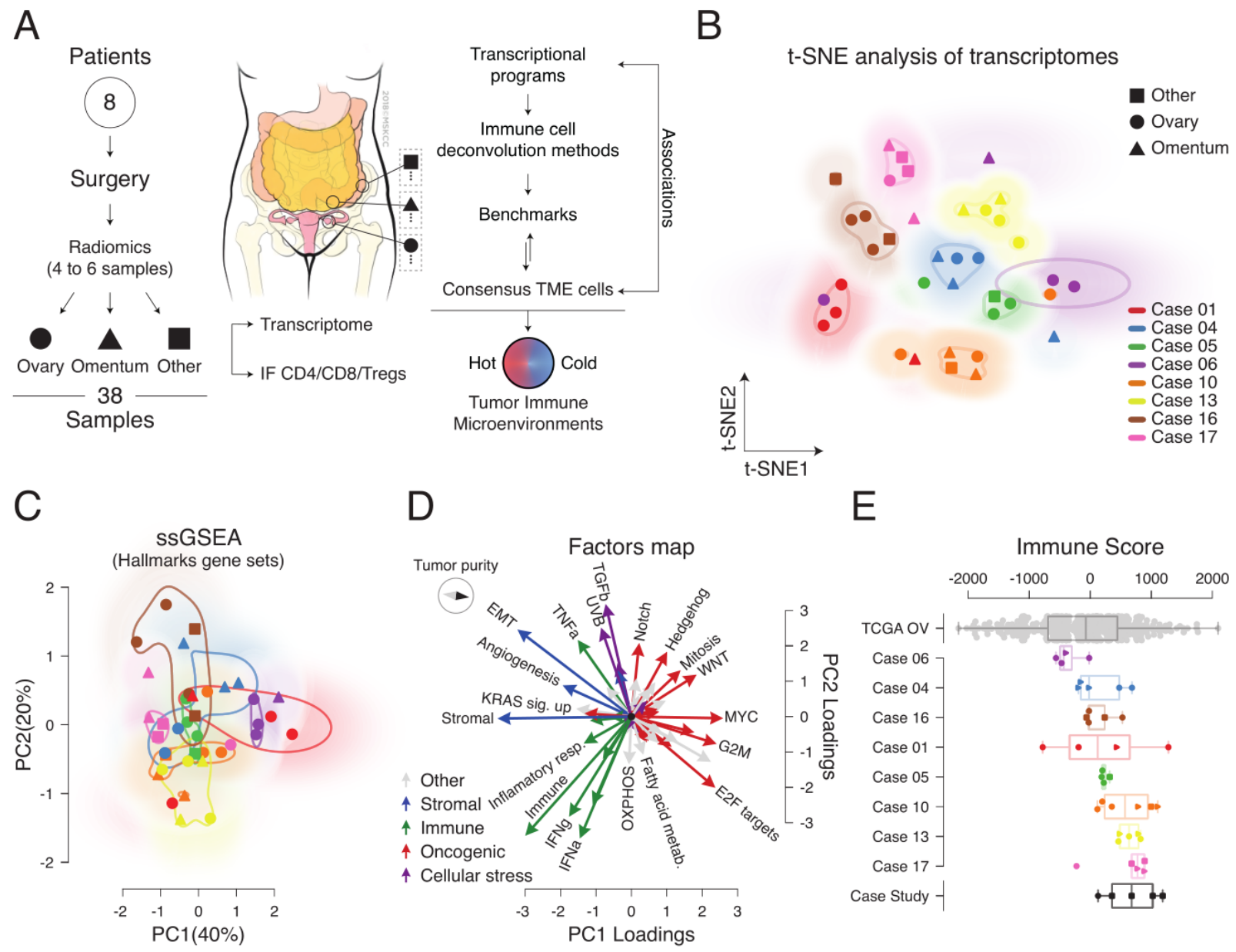

Figure 1: Immune signaling contributes to the majority of the transcriptional variance observed across multiple tumor samples from treatment-naive HGSOC patients. A) Flowchart of sample acquisition and analysis. Peritoneal metastases other than omentum were defined as "Other". B) t-SNE analysis of overall transcription profiles of multiple HGSOC tumor samples per patient. C) PCA of ssGSEA-based analysis of hallmark gene sets. D) Principal component feature loadings (magnitude and direction) of $\mathrm{C}$ are shown in the variables factor map. Vectors are colored according to a major biological classification of hallmark gene sets. Variation across classes in PC1 $\left(p=3.2 \mathrm{e}^{-16}\right)$ and PC2 $(p=0.02)$ after Kruskal-Wallis H-test (Figure S1). Directionality of ESTIMATE's tumor purity is represented with the map compass. E) ESTIMATE immune score across patients and samples. The Case Study samples were taken from (Jiménez-Sánchez et al. 2017). The bottom and top edges of the box plots indicate the 25th and 75th percentiles. 


\section{Co-existence of distinct tumor-immune microenvironments in treatment-}

\section{naive HGSOC}

To further characterize the tumor microenvironment of HGSOC, we performed multicolor immunofluorescence (IF) staining and quantification of CD4, CD8, and regulatory T cells (CD4+ FOXP3+) in at least 10 tumor regions excluding stromal areas in each sample leading to a compendium of 440 imaged and digitally quantified tumor regions (Figures 2A-B, S2, and Supplementary Table 2A). This multi-region and multi-site IF analysis shows that treatmentnaive HGSOC patients present variation in T cell infiltration in tumor deposits, ranging from less than $1 \%$ (e.g. patient 6 ) to $\mathrm{T}$ cells accounting for more than $10 \%$ of total cells in some areas (e.g. patient 01 and 10). Furthermore, some patients' tumor deposits demonstrated marked variation in T cell infiltration within the same tumor deposit across different habitats (e.g. patient 01). We then performed a linear mixed effects model analysis (see Methods and Supplementary

185 Table 2B) to statistically evaluate whether there is a difference of $\mathrm{T}$ cell infiltration between patients, between tumors of the same patients and between habitats within tumors. We found a remarkable difference in T cell infiltration across tumors within patients (Degrees of freedom=2, CD8 F-value=8758; CD4 F-value=58; Tregs F-value=657) and habitats within a tumor (Degrees of freedom=2, CD8 F-value=1184; CD4 F-value=2870; Tregs F-value=2216). Sites and habitats also showed an important level of interaction (Degrees of freedom=4, CD8 F-value=5915; CD4 F-value=4466; Tregs F-value=142). This systematic $T$ cell IF staining and computerized cell detection-counting confirm the variation in $\mathrm{T}$ cell infiltration across patients, across tumor samples within patients, within tumors and within habitats. Together with the transcriptome analysis, these data show that HGSOC is intrinsically characterized by the presence of 195 heterogeneous immune TMEs within patients and within tumors. 
A

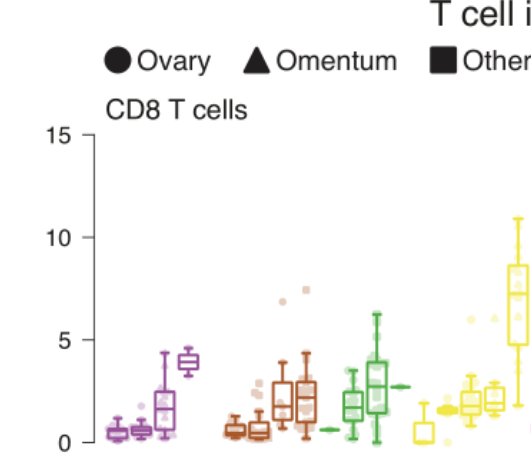

T cell infiltration
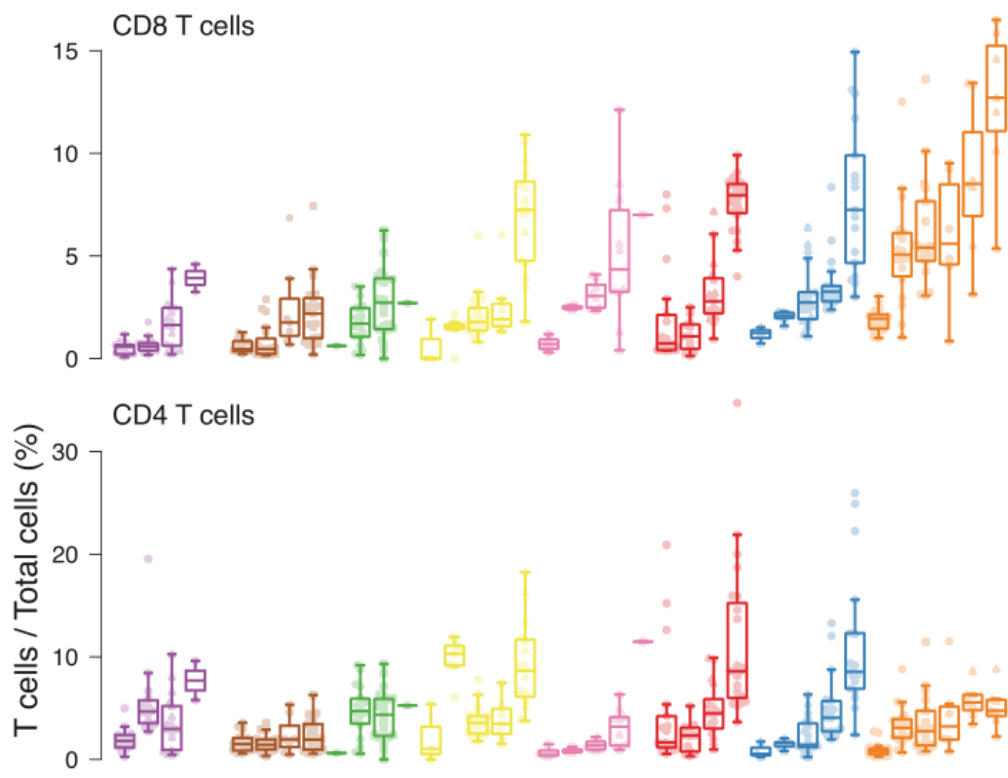

Tregs

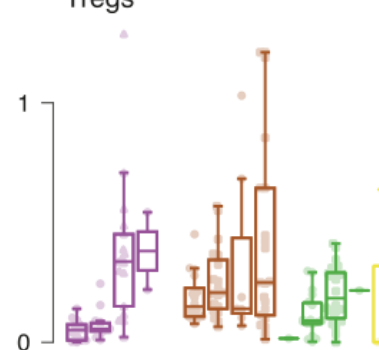

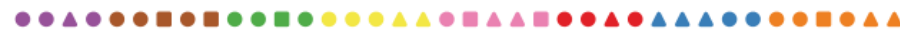

Habitat: $ү \beta \beta$ a $\beta \beta$ a a $\beta$ a a y a $\beta \beta$ ү $\beta$ ү $\beta$ a $\beta$ y a $\beta \beta$ y $\beta$ a a $\beta \beta$ a a $\beta$ y

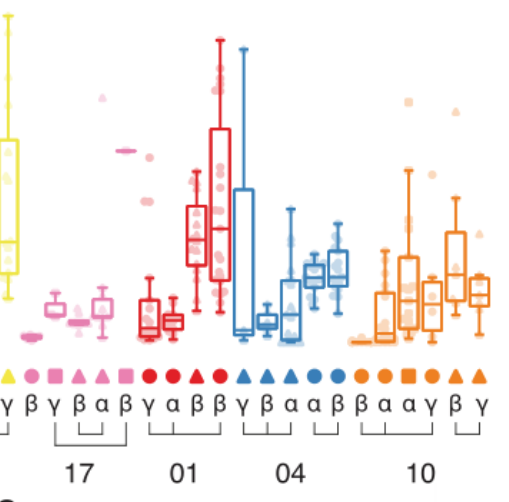

Immunofluorescent staining
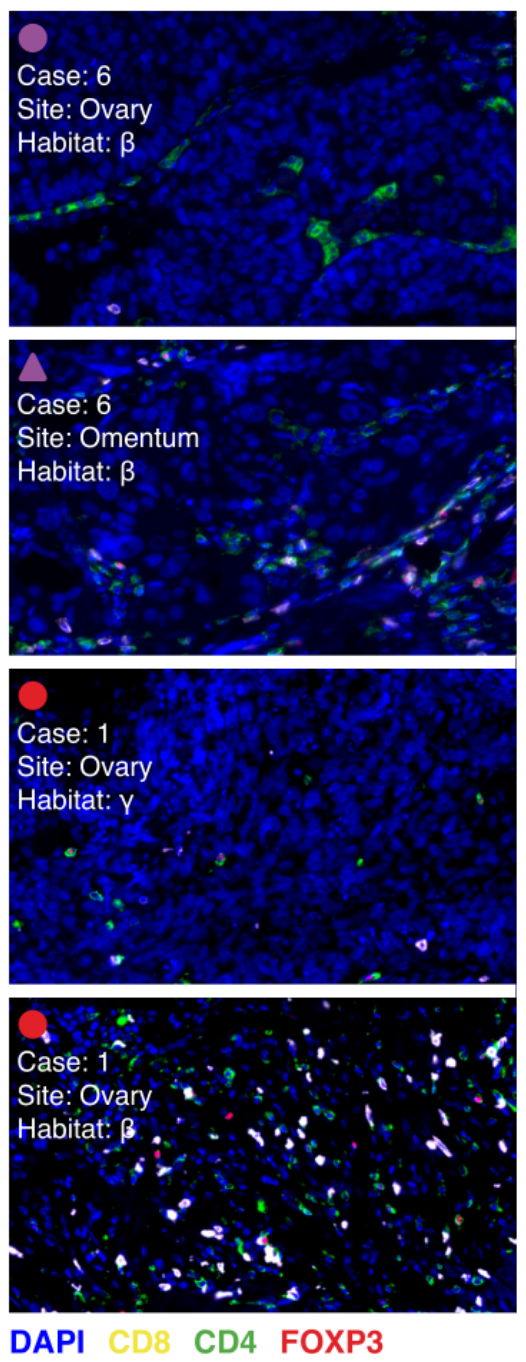

Figure 2: T cell infiltrate variation across patients, within patients, and within tumors. A) Multitumor sampling from 8 HGSOC patients are shown with each dot representing the percentage of T cell subsets in a quantified area within a given tumor section stained with multicolor IF for CD8, CD4 and FOXP3. Stromal areas were excluded based on H\&E stains. Patient cases are indicated by different colors. Anatomical sites of tumor deposits are indicated by different markers (circle, triangle and square). Habitats are defined by the greek letters $\alpha, \beta$ and $y$. Habitats from the same tumor are indicated by connecting lines. Boxplots are sorted according to the median of CD8 T cell infiltration across patients, sites and habitats accordingly. B) Representative images of panel A. 


\section{Consensus tumor microenvironment gene sets improve cell deconvolution}

\section{from bulk tumor mRNA}

To estimate the relative abundance of different cell types in an unbiased manner using bulk RNA data, various computational approaches have been developed during the last decade

210 (Finotello and Trajanoski 2018). However, a comparison that objectively evaluates the performance of these approaches against one another has not been conducted and no publicly available data sets have been generated to serve as ground truths thus far (Zheng 2017). Therefore, to test the performance of deconvolution methods, we performed a preliminary benchmark using the T cell IF quantification of the 440 regions from the 38 treatment-naive

215 HGSOC samples as the ground truth. We used ESTIMATE for total T cell infiltration (Yoshihara et al. 2013), and compared CIBERSORT (Newman et al. 2015), TIMER (B. Li et al. 2016), MCP-counter (Becht et al. 2016) and xCell (Aran, Hu, and Butte 2017) for cell type specific deconvolution. We also evaluated immune gene sets that were defined based on gene expression of sorted immune populations (Bindea et al. 2013), as well as immune gene sets

220 based on the Immunological Genome Project database (Davoli et al. 2017; Heng, Painter, and Immunological Genome Project Consortium 2008). Using ssGSEA (Hänzelmann, Castelo, and Guinney 2013), the Bindea et al. and Davoli et al. gene sets were used to calculate normalized enrichment scores (NES) of the corresponding cell types. To be able to evaluate the performance of ESTIMATE, which calculates a total immune score but does not deconvolute

225 immune cell types, total immune scores from the deconvolution tools were generated (see Methods). Total immune scores and deconvolution scores for CD4, CD8 and Tregs were correlated independently against the aggregated $\mathrm{T}$ cells and the corresponding cell type fractions (Figure 3A). Importantly, not all of the methods selected deconvolute CD4, CD8, and Tregs; however, for the methods that deconvolute all three cell types, none consistently 230 outperformed the other methods in this independent benchmark analysis. In addition, none of 
these methods was able to get a significant positive correlation with Tregs, which may indicate a lower-limit threshold of sensitivity of detection, since Tregs comprised, on average, less than $1 \%$ of cells in the tumor nests (Figure 2A, S2).

235 Since the cell type deconvolution methods were developed independently of each other, we reasoned that generating Consensus gene sets by including the genes that fall in the intersection of different cell types across the different tools could improve the cell deconvolution performance. Since not all methods deconvolute the same cell types, we focused on cell types that at least two methods deconvolute. We selected genes that overlapped between the 240 independent methods (intersection), and finally removed genes whose expression levels positively correlated with tumor purity using TCGA ovarian cancer samples as a reference (see Methods and Figure S3A). We correlated the sSGSEA NES of the Consensus TME cell gene sets against the fraction of $\mathrm{T}$ cells quantified, and observed that the Consensus gene sets consistently showed higher positive correlations than the individual methods. In addition, the

245 consensus Tregs NES was the only score with a significant positive correlation with the fraction of Tregs, suggesting a greater level of sensitivity obtained with the Consensus approach (Figure $3 \mathrm{~A}$, Spearman's rho $=0.26, q=6.2 \mathrm{e}^{-07}$ ).

To further benchmark the methods and the consensus signatures, we employed TCGA ovarian 250 cancer leukocyte methylation scores (Cancer Genome Atlas Research Network 2011), which is an independent and larger patient cohort. Leukocyte methylation measurements provide orthogonal means toward estimating immune infiltration in tumors, and have been shown to significantly correlate with histological purity estimates in primary HGSOC (Carter et al. 2012). Importantly, the leukocyte methylation signature was generated by comparing methylation

255 patterns between HGSOC tumors, normal fallopian tube samples and buffy coat samples of female individuals, making this data set ideal for benchmarking the deconvolution methods 
(Carter et al. 2012). We first performed a benchmark of all methods using the leukocyte methylation scores and the CD8 $T$ cell deconvolution scores (Figure 3B), as i) this subpopulation is a major component of infiltrating leukocytes, ii) all deconvolution methods

260 tested deconvolute CD8 T cells, and iii) overall the CD8 IF estimations were the best correlations (Figure 3A). Among the seven methods, the consensus CD8 gene signature correlated best with the CD8 $\mathrm{T}$ cell score (Figure $3 \mathrm{C}$, Spearman's rho=0.82 $p=3.9 \mathrm{e}^{-16}$ ). However, as the leukocyte methylation score does not count CD8 T cells exclusively, we then compared the different methods in an unbiased manner by fitting a multiple linear regression

265 model for each method using the leukocyte methylation score as a response variable and the different cellular scores as explanatory variables, followed by unsupervised nested variable selection (see Methods and Figure S3B). We compared the proportion of leukocyte methylation score variance that is explained by the unsupervised selected deconvoluted cells (adjusted Rsquared), as well as the relative quality of the models by considering goodness of fit and model 270 simplicity (see Methods). The Consensus gene signatures provided the highest adjusted Rsquared with fewer cell types selected (Adj. R-squared=0.73, $p<2.2 e-16$, Figure 3D left panel), as well as being selected as the simplest and most accurate model to explain leukocyte methylation (Figure 3D middle and right panels). Finally, the consensus gene signatures that the systematic unbiased analysis suggested best explained leukocyte methylation were cells 275 expected to be present in leukocyte infiltrates, with CD8 and NK cells accounting for the vast majority of the variation explained (Supplementary Table $3, \operatorname{CD} 8 p=1.74 \mathrm{e}^{-07}$, and NK cells $\left.p=1.79 \mathrm{e}^{-11}\right)$. In addition, we performed a sensitivity analysis of the leukocyte methylation benchmark, and the consensus was also the best method with the same cells explaining leukocyte methylation selected (Figure S3C and Supplementary Table 3). Together, these 280 benchmarks show a consistent improvement of leukocyte cell deconvolution provided by the Consensus gene sets in ovarian cancer samples. 
A

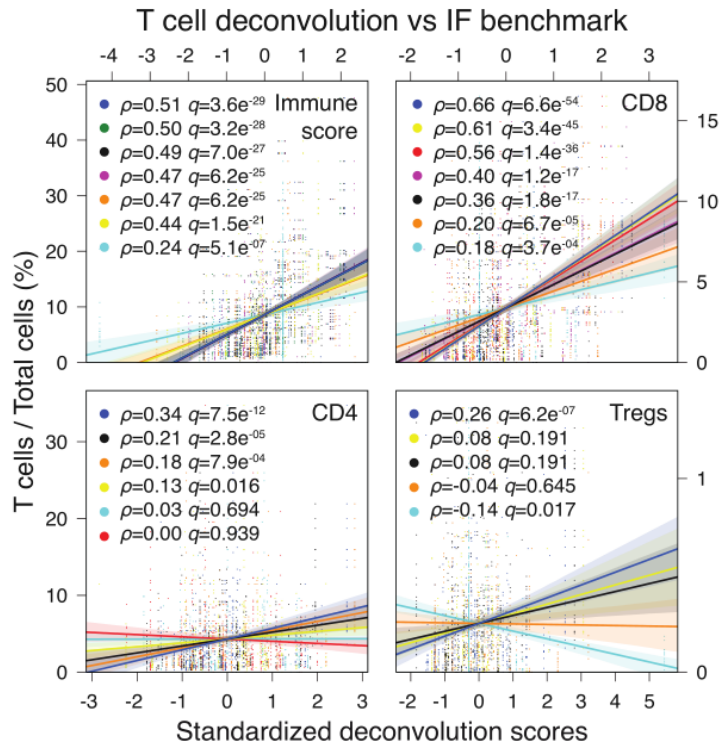

B

TCGA OV CD8 deconvolution benchmark

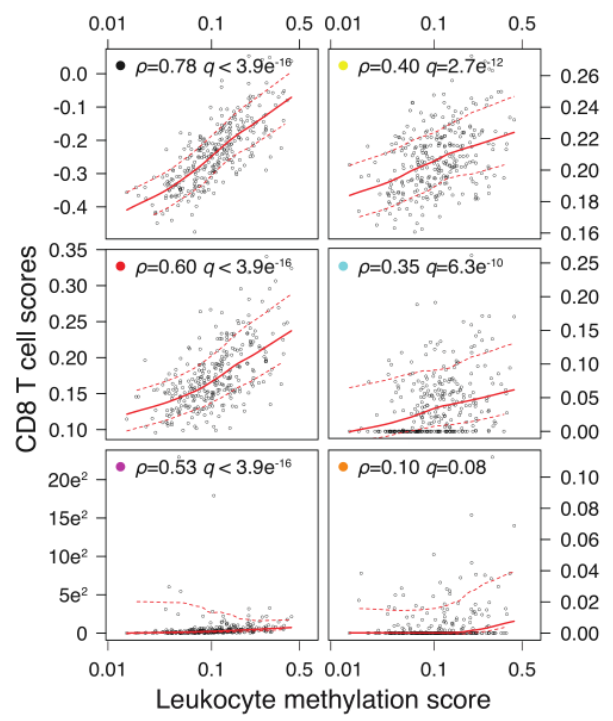

C

TCGA OV consensus CD8 deconvolution
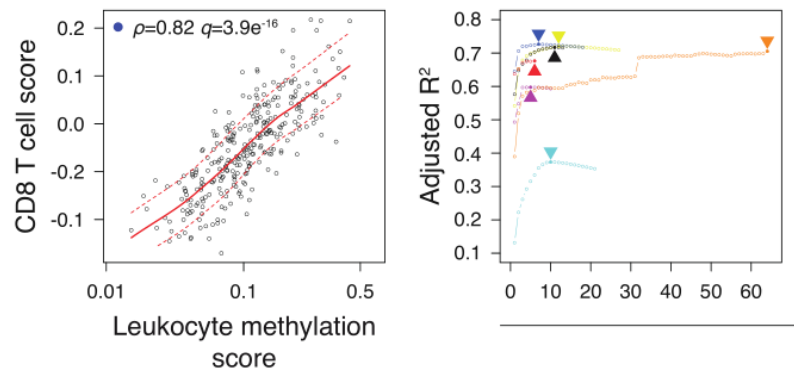

$\begin{array}{lllllll}0 & 10 & 20 & 30 & 40 & 50 & 60\end{array}$

Deconvolution methods

TCGA OV leukocyte deconvolution benchmark and model selection
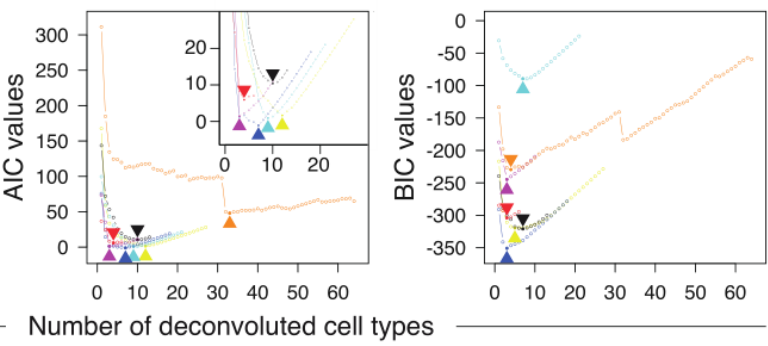

$\begin{array}{lllllll}0 & 10 & 20 & 30 & 40 & 50 & 60\end{array}$

es

CiBERSort estimate mCP counter ssGsea (Bindea)

ssGSEA (Davoli)

Figure 3: Consensus tumor microenvironment cell deconvolution method improves estimation of tumor infiltrating T cells and leukocytes. A) Spearman's rank-order correlations between percentage of T cells (immunofluorescent staining) and the corresponding deconvolution scores for each method using the treatment-naive ovarian cancer samples. Correlation coefficients $(\rho)$ and $q$-values are ordered from higher and more significant to lower and less significant. Deconvolution scores were standardized (z-score) to visually compare the different correlations in the same scale. IF immune score was calculated by adding up CD8, CD4, and Treg counts as an approximation, while deconvolution immune scores were calculated according to each deconvolution tool (see Methods). B, C) TCGA ovarian cancer Spearman's rank-order correlations between CD8 T cell deconvolution scores and leukocyte methylation scores. D) Multiple linear regression analysis using leukocyte methylation score as response variable, and 
deconvoluted cell types as explanatory variables (see Methods). Adjusted $\mathrm{R}^{2}$, Akaike information criterion $(\mathrm{AIC})$, and Bayesian information criterion $(\mathrm{BIC})$ values were calculated to compare goodness of fit and

295 model simplicity. Arrowheads indicate best model for each method. Inset in the AIC panel shows a magnification of the best ranked models.

\section{Tumor microenvironment cell deconvolution and molecular comparison of}

\section{high and low purity treatment-naive HGSOC}

Having generated our own robust method for immune cell deconvolution, the Consensus

300 method, we applied the Consensus gene sets to the treatment-naive HGSOC transcript data to systematically assess if specific transcriptional programs were associated with variability in immune infiltration. We first visualized the variation across samples using the NES of deconvoluted Consensus gene sets of cells (Figure 4A, S4A). The gene sets explaining most of the variation were cytotoxic, NK cells and fibroblast being negatively correlated with tumor

305 purity; while endothelial, monocytes and B cells positively correlated with tumor purity (Figure 4B, S4B). The NES of deconvoluted cells of this cohort were comparable to the NES obtained in the TCGA ovarian cancer data set (Figure S5). Interestingly, the cells with highest NES in most samples were fibroblasts, highlighting the intrinsic low-immunogenic nature of HGSOC (Wang et al. 2016).

To investigate genes associated with tumors with high cellularity (pure tumors), we used the median tumor purity of the cohort to classify high and low purity tumors (see Methods), and performed a differential expression analysis leveraging sample-patient dependency (i.e. considering that multiple tumors come from the same individual) to increase statistical power.

315 As expected, genes related to immune activation were significantly highly expressed in low purity tumors, but only eleven genes were significantly highly expressed in the purer tumors compared to the lowly pure ones (Figure 4C). Gene ontology analysis (GO) showed that genes 
with significant higher expression in low purity tumors are significantly enriched in leukocyte proliferation and activation GO biological processes (Figure 4D), whereas no significant GO 320 enrichment was found with the genes significantly highly expressed in pure tumors. The genes with significantly higher expression in pure tumors have been implicated in transcription [CPSF6 (Rüegsegger, Blank, and Keller 1998), FOXJ3 (Landgren and Carlsson 2004)], cellular growth [HOMER2 (Tu et al. 1998; Xiao et al. 1998), REPS1 (Cantor, Urano, and Feig 1995; Hu and Mivechi 2003)], glucose transport [MFSD4B (Horiba et al. 2003)], mitocondrial generation 325 [PTCD3 (Davies et al. 2009)], aberrant proliferation [YBX1 (Frye et al. 2009; Weidensdorfer et al. 2009)], ovarian cancer initiation and progression [ACP6 (Hiroyama and Takenawa 1999; Fang et al. 2002), PARP2 (Amé et al. 1999; Gunderson and Moore 2015)] and TGF-beta signaling downregulation [FAM60A (Muñoz et al. 2012; Smith et al. 2012)], which consequently can directly and indirectly promote tumorigenesis through TME immunosuppression (Colak and ten Dijke 2017; Tauriello et al. 2018; Mariathasan et al. 2018). 
A

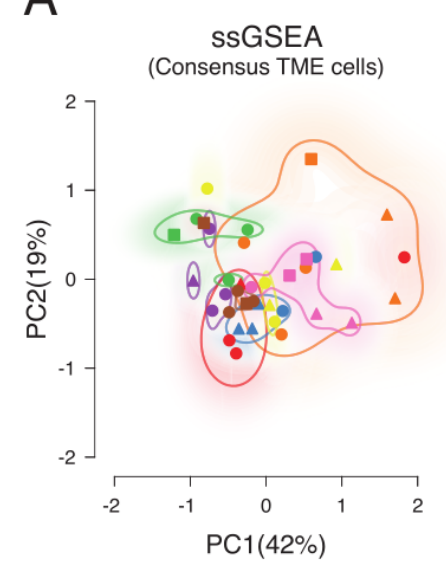

$\mathrm{D}$

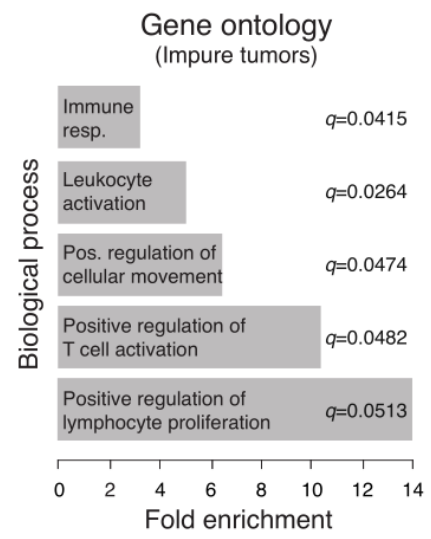

B

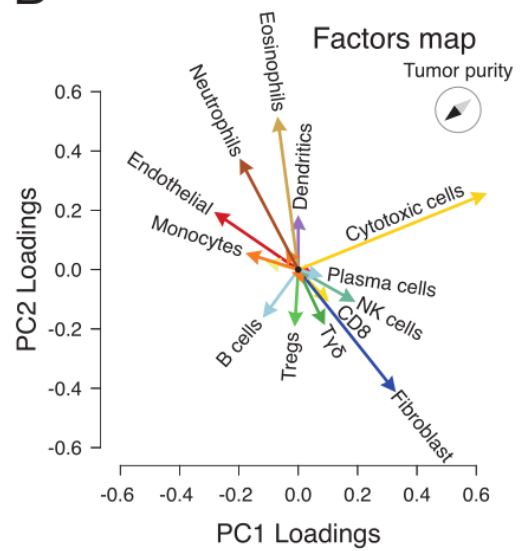

$E$

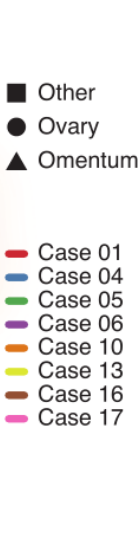

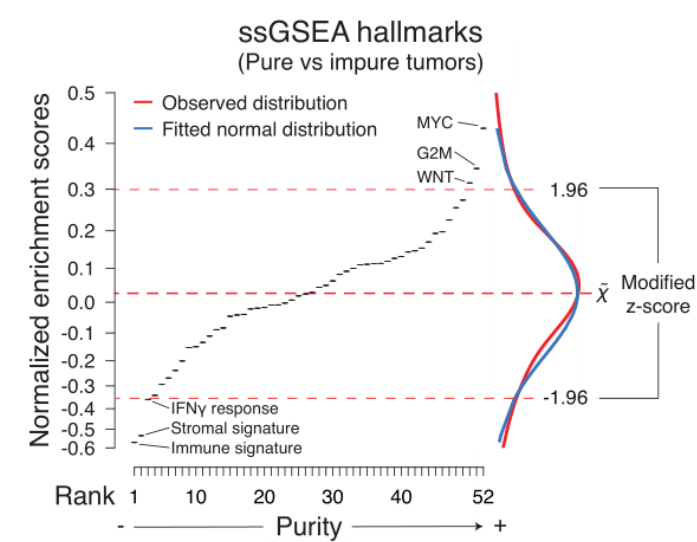

C

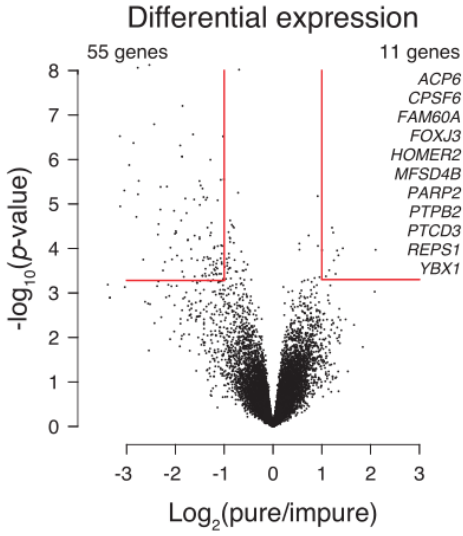

$\mathrm{F}$

SsGSEA consensus TME cells (Pure vs impure tumors)

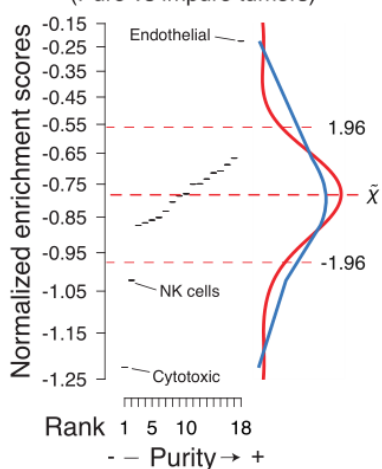

Figure 4: Unbiased analysis of tumor microenvironment heterogeneity in treatment-naive HGSOC tumors. A) PCA of ssGSEA-based analysis using the consensus deconvoluted cell gene sets. B) Principal component feature loadings (magnitude and direction) of A. Vectors are colored according to cell types, for example monocytes and macrophages M0, M1, M2 (orange), B cells and plasma cells (light blue), and CD8 and cytotoxic cells (yellow). C) Differential expression analysis of high purity and low purity classified tumors using the median purity score of the cohort as a cutoff (see Methods). Vertical red lines indicate $+/-1$ fold change of gene expression, and the horizontal line indicates the corresponding $0.05 q$-value on the y-axis. D) Gene ontology analysis of significantly highly expressed genes on low purity tumors. Significantly highly expressed genes in pure tumors are not significantly over represented in any gene ontology biological process. E, F) ssGSEA analysis of differentially expressed genes using hallmarks and consensus deconvoluted cell gene sets, respectively (see Methods). Gene sets on the xaxes were ranked according to their normalized enrichment score (Supplementary Tables 4A-B). Higher 
normalized enrichment score are indicative of higher purity scores. Dashed red lines indicate median and $345+/-1.96$ median absolute deviations (modified z-score) to define outliers. Marginal density plots of observed and values fitted to a normal distribution are shown.

To further investigate which molecular signaling pathways are more highly enriched in pure tumors, we performed SSGSEA using the adjusted $p$-values and changed the sign, positive or 350 negative, according to the differential expression direction (see Methods). As expected, immune and stromal signatures were highly enriched in low purity tumors, in addition to IFN-gamma response. In contrast, Myc and Wnt signaling appeared to be highly enriched in pure tumors, both of which have been previously associated with immune exclusion in pre-clinical models of lung cancer (Kortlever et al. 2017) and melanoma (Spranger, Bao, and Gajewski 2015;

355 Spranger et al. 2016; Spranger, Bao, and Gajewski 2014), respectively (Figure 4E). Not surprisingly, the proliferation related hallmark G2M was highly enriched in pure tumors. Of note, little or no overlapping between the G2M, Myc and Wnt hallmark gene sets was observed (Figure S4C and Supplementary Table 4A; 6 out of 258 genes overlapped between G2M and Myc, and 2 out of 242 genes overlapped between G2M and Wnt signaling gene sets).

360 Considering the TME, cytotoxic and NK deconvoluted cells were preferentially enriched in low purity tumors, whereas endothelial cells were highly prevalent in pure tumors (Figure 4F). Since all genes in the cytotoxic gene set are included in the CD8 and NK cell gene set, this suggests that a particular activation of NK cells is more prominent in low purity tumors (Figure S4C), whereas pure tumors only showed enrichment of endothelial cells (Supplementary Table 4B).

365 These observations suggest that Myc and Wnt signaling gene set enrichments in pure tumors could be considered at least partially independent of tumor proliferation, and may also contribute to immune cell exclusion as suggested by other studies (Spranger and Gajewski 2018). 


\section{Chemotherapy induces immune activation in HGSOC}

To investigate the effect of chemotherapy on the TME and evaluate if there is a confounding effect of the intra-patient TME heterogeneity described above, we studied the transcriptome of site-matched $(n=18)$ and site-unmatched $(n=38)$ primary and disseminated tumors before and after treatment with neoadjuvant platinum and taxane chemotherapy in 28 HGSOC patients (Figure 5A, Supplementary Table 5A-B). Using t-SNE dimensionality reduction

375 on the whole transcriptomes, we found that treated and untreated samples cluster separately (Figure 5B), in contrast to the treatment-naive samples that cluster in a patient-specific manner (Figure 1B). Using the ssGSEA NES of the hallmark gene sets of site-matched and siteunmatched samples, we observed that treated and untreated sample groups were separated by the two first principal components with $63 \%$ and $50 \%$ of variation in site-matched and site-

380 unmatched groups, respectively (Figures 5C and S6A-B). Both site-matched and siteunmatched groups showed that oncogenic and immune/stromal hallmarks contributed significantly to the variation explained by the first principal components (Figure S6C-D). However, only site-matched PC1 reached statistical significance after paired comparison between pre- and post-NACT samples (Figure S6E-F), while also explaining more than $50 \%$ of

385 the variation in the site-matched samples (Figure S6A-B). Interestingly, cellular stress related pathways were more enriched in site-unmatched post-NACT than site-matched samples, potentially reflecting the cellular stress generated by therapy, whereas in site-matched samples, immune related pathways dominate the variation signal (Figures 5C, S6C-D). In addition, Wnt and Myc signaling showed a clear negative association to immune related gene sets in the sitematched samples, whereas no clear clustering contribution of Wnt and Myc gene sets is discernible in the site-unmatched samples (Figure 5C). 

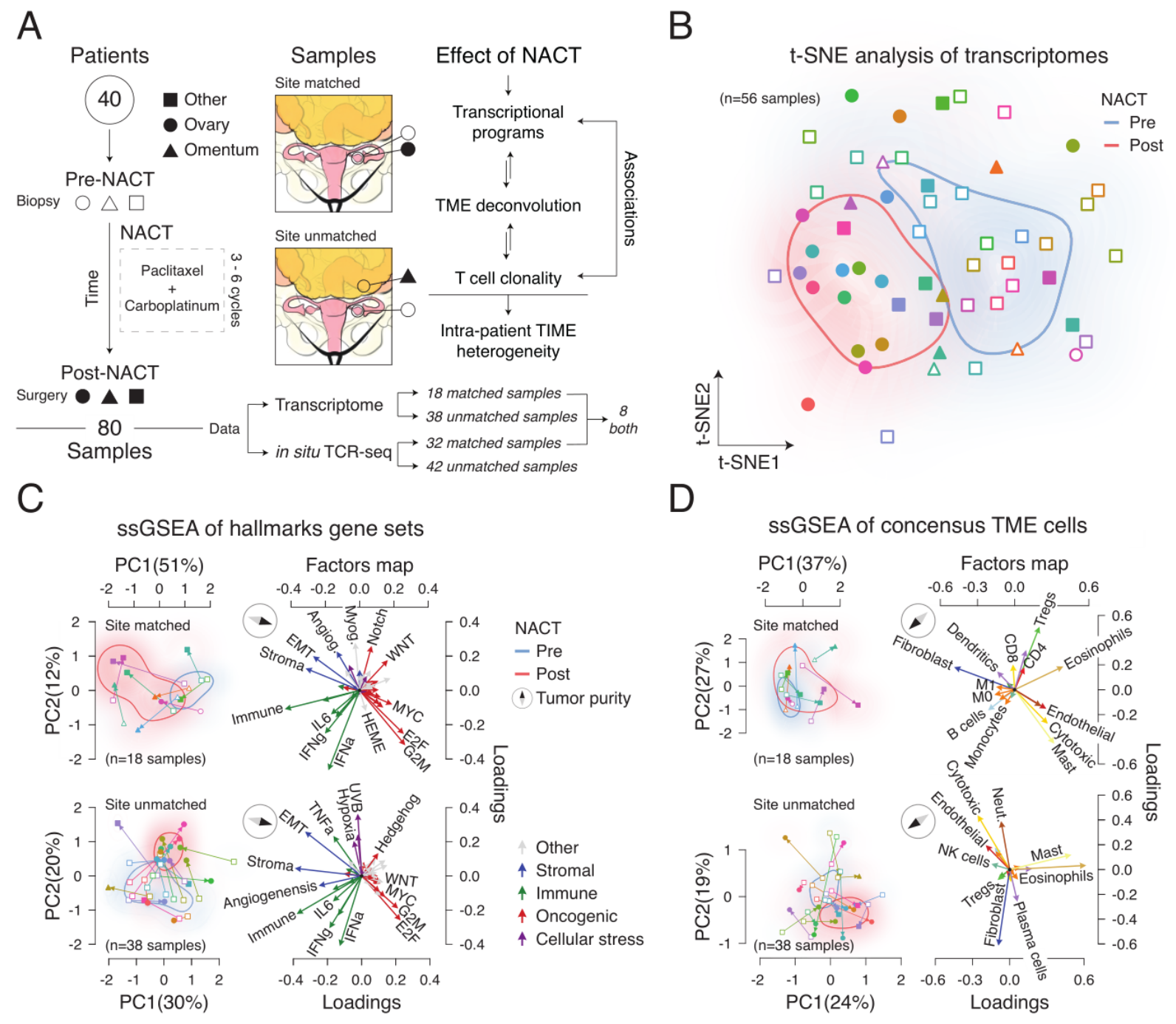

Figure 5: Unbiased signaling pathway and tumor microenvironment cell decomposition analysis

of chemotherapy treated HGSOC site-matched and unmatched tumor samples. A) Flowchart of sample acquisition, clinical study design, and analysis. TME: Tumor microenvironment. B) t-SNE analysis of overall transcription profiles of multiple HGSOC tumor samples per patient. C, D) PCA and principal component feature projections (magnitude and direction) of sSGSEA-based analysis of hallmark gene sets and consensus tumor microenvironment (TME) cells respectively. Arrows in the principal component space indicate pre- to post-NACT directionality. Hallmark gene set vectors are colored according to a major biological classification. Angiog: Angiogenesis, Myog: Myogenesis. Consensus TME vectors are colored according to cell types. Neut: Neutrophils. 
405 We then deconvoluted the TME cellular mixtures to investigate which cells were differentially present in the pre- and post-treatment tumors. Pre- and post-treatment samples also clustered separately both in site-matched and site-unmatched samples, with the first principal components accounting for $64 \%$ and $43 \%$ of variation explained, respectively (Figures 5D, S7AB). Eosinophils and cytotoxic cells showed a negative association with tumor purity in site-

410 matched samples, in contrast to fibroblasts, B cells and macrophages (Figures 5D, S7C). Interestingly, the only principal components that were significantly different between pre- and post-NACT samples were the first two principal components of the site-matched tumors (Figure S7E-F).

\section{Tumor-immune microenvironment intra-patient heterogeneity masks}

415 chemotherapy induced immune activation effect

To directly evaluate differences between pre- and post-treatment samples, we performed an exploratory data analysis leveraging the possibility of performing paired comparisons using the hallmark and consensus TME gene set NES independently for sitematched and site-unmatched samples (Figure 6A). Site-matched samples showed a clear

420 increase of immune pathways and consensus TME gene sets in post-treatment samples, while site-unmatched samples showed an increase of cellular stress pathways reflecting cellular and metabolic stress after cytotoxic drug exposure, but no difference of consensus TME gene sets was detected in the site-unmatched cohort. Since we observed that cytotoxic and NK cell Consensus gene sets were mainly enriched in treatment-naive low purity tumors (Figure 4F), 425 and it is known that CD8 T cells play a crucial role in ovarian cancer recurrence and overall survival (L. Zhang et al. 2003), we performed a multivariate Student's t-statistic hypothesis test using cytotoxic, NK and CD8 T cell Consensus gene sets. We compared the difference between these 3 Consensus gene sets in pre- and post-NACT site-matched and site-unmatched samples 
(Figure 6B), and observed a significant increase of these immune cell types upon chemotherapy

430 in the site-matched samples $(p=0.0034)$, but no difference in the site-unmatched samples $(p=0.92)$. To further evaluate $\mathrm{T}$ cell infiltration and activation between pre- and post-NACT samples, we performed in situ TCR sequencing. Since T cell activation leads to clonal expansion of particular T cell clonotypes, TCR clonality measures can be used as a surrogate for T cell activation upon specific (neo)antigen recognition (Pielou 1966; Kirsch, Vignali, and

435 Robins 2015; Jiménez-Sánchez et al. 2017a). TCR clonal expansion was significantly higher in post-NACT site-matched samples (Figure $6 \mathrm{C}, p=0.001$ ), but no significant difference was observed in site-unmatched samples $(p=0.2)$. T cell fraction was also significantly higher in postNACT site-matched samples ( $p=0.03$ ), while a slightly lower T cell fraction was observed in siteunmatched post-NACT tumors, potentially as a result of the variability of immune infiltration

440 between omentum metastases (pre-NACT biopsies) and primary tumors (post-NACT debulking surgery). To test whether the biopsy intervention could be a confounder and lead to an immune activation per se, we compared post-NACT site-matched and post-NACT site-unmatched TCR clonal expansions, since the post-NACT site-unmatched tumors were not originally biopsied. No significant difference was observed in TCR clonal expansion $(p=0.67)$, suggesting that $\mathrm{T}$ cell

445 clonal expansion was independent of biopsy treatment and likely induced by NACT. However, a significant increase of T cell density was observed in site-matched compared to site-unmatched post-NACT tumors $\left(p=3.59 \mathrm{e}^{-05}\right)$, potentially suggesting that wound healing after the biopsy procedure could increase the influx of $\mathrm{T}$ cells. Together, these results provide evidence that neoadjuvant chemotherapy induces an immune activation in the local TME of HGSOC, and that 450 intra-patient inter-site TME heterogeneity can obscure this clinically relevant observation among tumor deposits within patients. 

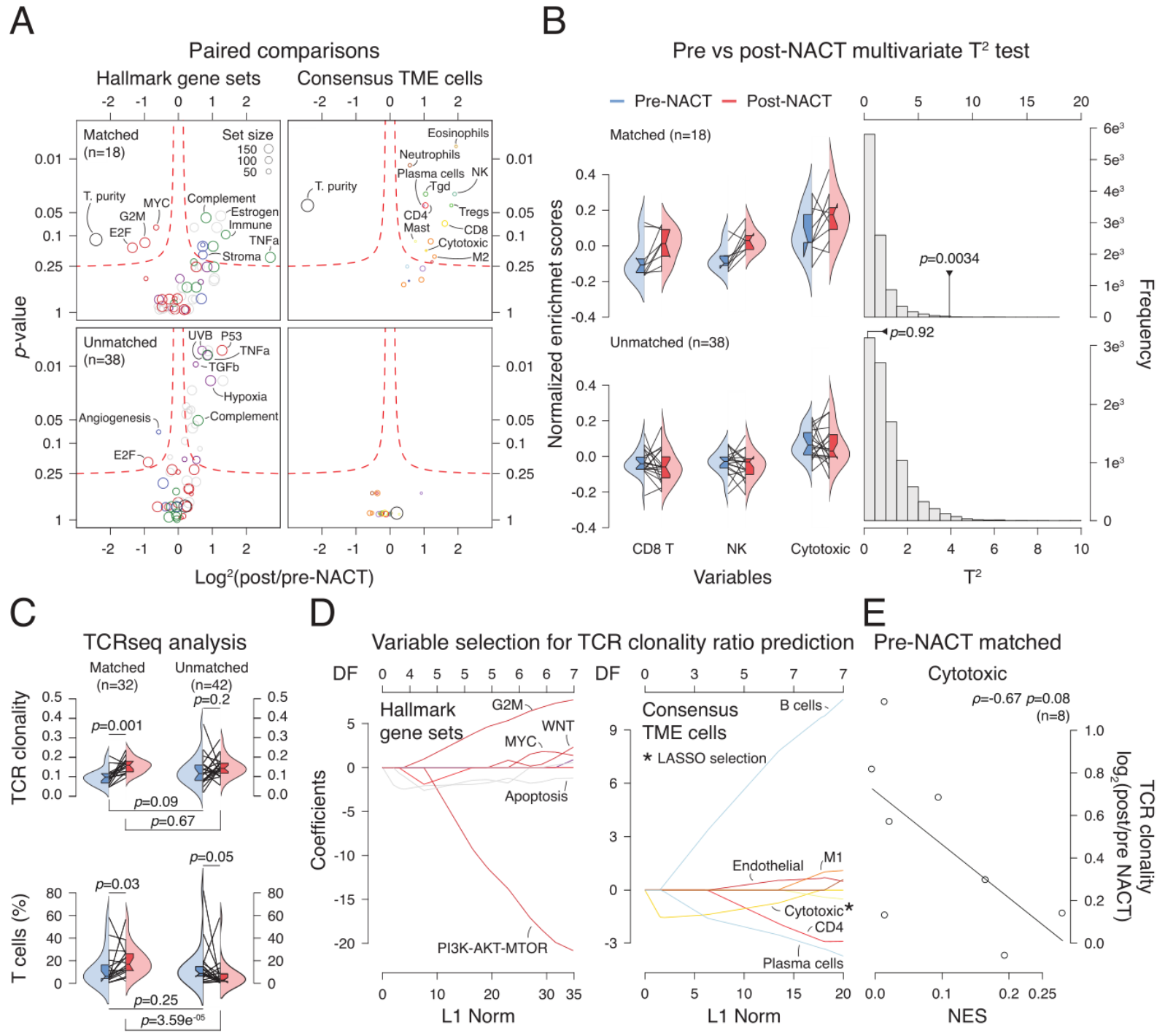

D

Variable selection for TCR clonality ratio prediction Pre-NACT matched
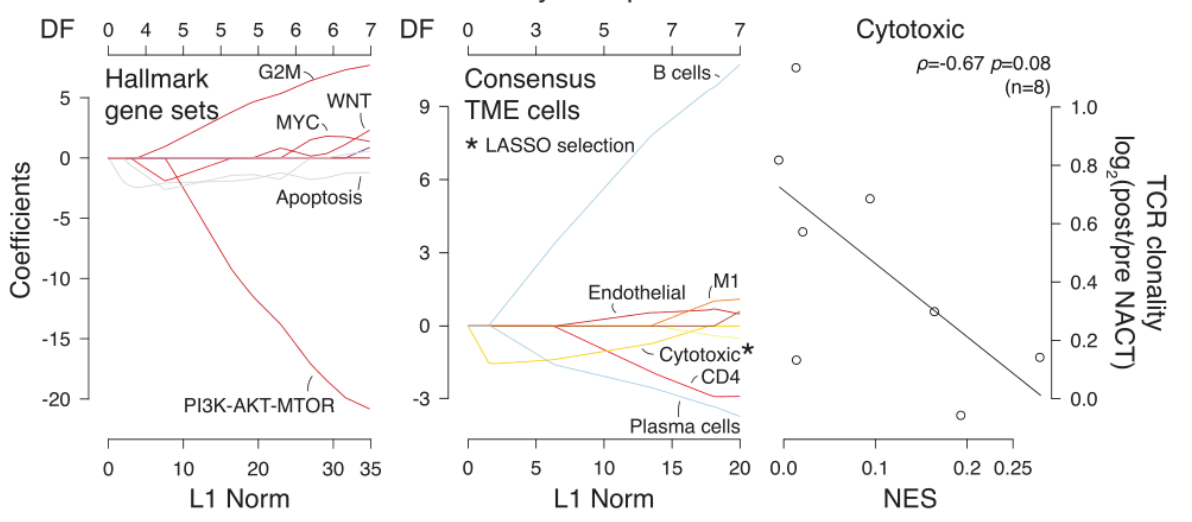

Figure 6: Immune activation induced by neoadjuvant chemotherapy is evident in site-matched but

455 not site-unmatched sample analysis. A) Exploratory pre/post NACT paired comparisons of hallmark gene sets and consensus TME deconvoluted cells (see Methods). B) Multivariate $\mathrm{T}^{2}$ tests comparing pre and post-NACT CD8 T cells, NK cells, and cytotoxic NES together. C) Comparisons of TCR productive clonality (top), and percentage of productive T cells (bottom) between pre and post-NACT site-matched and site-unmatched samples. Paired and unpaired tests were used accordingly. TCR clonality is expressed as 1-entropy with values near 1 representing samples with one or a few predominant TCR rearrangements, while values near 0 represent more polyclonal samples $\mathrm{D}$ ) Least absolute shrinkage and selection operator (LASSO) regression analysis using the pre-NACT matched ( $\mathrm{n}=8$ samples) hallmark 
and consensus TME cell NES as explanatory variables, and the $\log _{2}$ of the TCR clonality ratio of post/preNACT as response variable (see Methods). DF: Degrees of freedom. The variables selected by the

465 LASSO regression are indicated with an asterisk. E) Spearman's rank correlation of pre-NACT cytotoxic NES and $\log _{2}$ of the post/pre-NACT TCR clonality ratio.

Finally, we investigated whether hallmark pathways or consensus TME gene signatures calculated from the pre-treatment samples could explain the increase of TCR clonality upon 470 neoadjuvant chemotherapy in site-matched samples $(n=8$, only 8 pre-treated samples with both gene expression and TCRseq data were available, see Figure 5A, Supplementary Table 5B). To perform the analysis in an unbiased manner, we employed least absolute shrinkage and selection operator (LASSO) regression analysis with the change of TCR clonality before and after NACT as a response variable (see Methods). The hallmark pathways that potentially have 475 predictive value with positive association were the hallmarks G2M checkpoint, Myc and Wnt signaling and UV response, while apoptosis and PI3K-AKT-MTOR showed a negative association with TCR clonality increase (Figure 6D). We then performed the same analysis using the consensus TME gene signatures, where B cells, M1 macrophages and endothelial cells showed a positive association with TCR clonal expansion, while cytotoxic, CD4 and 480 plasma cells showed a negative association. In addition, the LASSO analysis selected the consensus cytotoxic signature as relevant for explaining T cell clonal expansion upon NACT, and a correlation analysis supported this association (Figure 6E). Post-selection inference, taking into account for the uncertainty of the model selection and multiplicity, corroborated that the pre-NACT NES of the Consensus cytotoxic gene set is a promising variable to explain T cell 485 clonal expansion upon NACT in these eight samples ( $p=0.096$, see Methods). Overall, these results show that chemotherapy induces a T cell activation in HGSOC in site-matched samples but not in site-unmatched samples, further suggesting that different local immunemicroenvironments play a role in the response to chemotherapy treatment. Also, pre-treatment 
490 chemotherapy, while tumors with higher infiltration levels do not have such a high clonal expansion, an observation that could not be addressed in unmatched tumor samples, due to the intra-patient tumor-immune heterogeneity. This highlights the potential confounding effects that intra-patient tumor-immune heterogeneity imposes on comparing tumors not only from different patients but also from different sites within the same patient.

\section{DISCUSSION}

Despite advances in surgical approaches, chemotherapy and targeted therapies, the prognosis for patients with high grade serous ovarian cancer remains poor, with the near-inevitable development of resistance to systemic therapy. Genetic and molecular analyses of asynchronous and disseminated tumors within patients have recently started to shed light on

500 tumor clonal dynamics and evolutionary properties of different tumor types (Johnson et al. 2014; Yates et al. 2015; McPherson et al. 2016); however, the extent of TME heterogeneity in advanced HGSOC has only begun to be revealed (Jiménez-Sánchez et al. 2017; A. W. Zhang et al. 2018). We explored the main sources of variation in the transcriptomic space among treatment-naive samples and detected that transcriptomic pathway heterogeneity is mainly

505 explained by presence or absence of immune and stromal cells. Importantly, the degree of immune signature variation within patients was similar to the extent we observed in a case study of metastatic HGSOC, where different tumor immune microenvironments were associated with clinical outcome. In the index case, tumors with high immune related pathways regressed and presented evidence of $\mathrm{T}$ cell activation, while immune excluded tumors progressed (Jiménez-

510 Sánchez et al. 2017). In the present study, all patients presented at least one tumor with low immune infiltration, suggesting that HGSOC is characterized by microenvironmental niches, which could underlie primary and acquired resistance to therapies (Wang et al. 2016; Sharma et 
al. 2017; Hirata et al. 2015). Through tissue image analysis, we captured immune signature differences and variation of $T$ cell infiltration within tumors which was confirmed by

515 immunofluorescence staining of T cells. Taken together, the transcriptional, imaged-based and immunofluorescence analyses show that TME heterogeneity is an intrinsic feature of HGSOC, which spans across patients, tumors within patients and within tumors. Furthermore, we found that intra-patient TME heterogeneity can mask the immune activation generated by treatment with cytotoxic chemotherapy. These analyses provide firm evidence that the TME affects the 520 extent of immune activation generated upon treatment with chemotherapy.

Since the TME can constrain or foster tumor progression, targeting the TME represents a promising alternative to complement therapeutic strategies (Hansen, Coleman, and Sood 2016). However, the degree to which TME heterogeneity is driven by stochastic, cellular or molecular 525 processes is not well understood, and little is known on the potential mechanisms behind TME spatial heterogeneity in HGSOC. Previous unbiased immune deconvolution studies have estimated relative abundances of immune cells in ovarian cancer tumors (Newman et al. 2015), and calculated survival associations (B. Li et al. 2016); however, no objective benchmarking has been performed to date, and discordant results make it difficult to judge these observations (B.

530 Li, Liu, and Liu 2017; Newman et al. 2017; Zheng 2017b). We integrated data from different deconvolution methods and generated a consensus approach which consistently improved the predictions on our data sets and in TCGA ovarian cancer leukocyte methylation data. This showed that cytotoxic and NK cells are the major populations present in low purity tumors, while endothelial cells are the main TME cell type in high purity tumors. A previous pan-cancer 535 analysis showed negative correlations between somatic copy number alterations (SCNA) and deconvoluted immune infiltrates, and that NK cells and CD8 T cell receptor pathway were the most differentially abundant immune factors between tumor samples with high versus low SCNA (Davoli et al. 2017). Despite these associations, the molecular mechanisms underlying this 
540 Interestingly, NK cell infiltration appeared to be significantly higher in tumors with low compared to high SCNAs in HGSOC (Davoli et al. 2017), and our results also showed that NK cells were more enriched than CD8 and CD4 T cell infiltration in low purity tumors. Together, these results indicate that NK cells could be a potential TME cellular target in HGSOC, and further investigations would be required to validate this.

Transcriptional signatures can be highly informative as diagnostic/prognostic resources, as well as provide insights on mechanistic underpinnings (Burel and Peters 2018). In this study we took advantage of the availability of having multiple tumors from the same patients and performed differential expression analysis between high and low purity tumors. Pathway analysis of the

550 differentially expressed genes showed that Wnt and Myc signaling pathways were more prevalent in purer tumors, consistent with emerging data in HGSOC and other tumors and models (Gounari et al. 2002; Damsky et al. 2011; Spranger, Bao, and Gajewski 2015; Spranger et al. 2016; Sridharan et al. 2016; Spranger et al. 2017). In addition, different studies have observed association between loss of $p 53$ function and decrease of NK cell infiltration in mouse 555 models (Xue et al. 2007) and T cell infiltration human breast cancers (lannello et al. 2013) In fact, missense or nonsense mutations in p53 are the earliest and almost ubiquitous (96\%) alterations in HGSOC (Ahmed et al. 2010; Cancer Genome Atlas Research Network 2011). Overall, these different lines of evidence help to clarify why HGSOC is intrinsically nonimmunogenic: beyond the low somatic missense mutation load and the high SCNAs, the 560 intrinsic oncogenic signaling of HGSOC seem to shape the TME and hinder immune infiltration of T cells and NK cells.

There are significant potential clinical implications from understanding the effect of chemotherapy on the TME and the molecular drivers of the heterogeneity observed, as novel 
565 combination therapies or changes in timing of treatments have the potential to improve outcomes (Patel and Minn 2018). A previous study investigated the effect of NACT on the activation of CD8, CD4 and Tregs in HGSOC, as well as systemic levels of cytokines (Böhm et al. 2016). This study found that patients who had good responses to NACT had a decrease of Tregs after treatment compared to poor responders. In general, there was also a trend towards 570 higher cytolytic activity in tumors after NACT despite failing to detect significant changes on CD8 T cell counts (Böhm et al. 2016). Using our unbiased approach and using the consensus deconvolution method, we observed an increase of cytotoxic immunogenic activity after NACT in matched tumor samples but not in site-unmatched samples from the same patient. Similarly, employing TCRseq, we found a significant increase in T cells and TCR clonality in matched 575 samples, but no significant difference was detected in unmatched pairs. Comparing post-NACT site-matched and post-NACT site-unmatched samples indicated that the observed change in TCR clonal expansions was driven by chemotherapy and not by the biopsy itself, although we cannot formally exclude potential immunogenic effects that the biopsy procedure may have in a neoadjuvant setting. Together, these results show a clear, confounding effect that spatial TME 580 heterogeneity can cause.

Having unmasked the immune activation generated by NACT, we used the matched samples to investigate the factors in pre-treated samples that influence TCR clonal expansion induction upon NACT. Interestingly, cytotoxic cells showed a significant negative association with TCR 585 clonal expansion, and the PI3K-AKT-MTOR pathway, which is part of the TCR signaling cascade, also showed a strong negative association. Conversely, Wnt and Myc signaling appeared as positively associated with TCR clonal expansion. Together, these results suggest that tumors with low levels of infiltration have a higher potential for T cell activation upon NACT than tumors with a previous immune presence. We hypothesize that this could be due to an 590 already exhausted immune TME generated as a consequence of chronic immune and tumor 
interaction, while immune excluded tumors present a fresh environment where $\mathrm{T}$ cells can become active and expand. Importantly, our results point towards NK cells being similar or more activated after NACT, suggesting another potential therapeutic cellular target for combination therapy. Finally, this conclusion could only be drawn only when intrapatient spatial TME 595 heterogeneity was controlled for, again highlighting the necessity to take TME heterogeneity into consideration in translational studies and in clinical applications.

Here we have presented how TME heterogeneity is a common feature of HGSOC and how that can affect the interpretation of translational studies. We have sought to uncover molecular and 600 cellular mechanisms behind intra-patient and intra-tumor TME heterogeneity. However, there are critical limitations to consider. Disentangling the actual mechanisms using human tumor samples represents a formidable challenge since tissue samples are limited, inter-patient variability is prominent and mechanistic experimental validation is prohibitive. Given these constraints, this study is descriptive in nature and relies heavily on observations derived by 605 independent studies using murine tumor models to propose suitable explanations. Despite this main limitation, our unbiased analysis of human tumors not only complements experimental studies, but also provides new hypotheses to further explore in a pre-clinical and clinical settings. Another major limitation is the small number of samples available compared to the large parameter space to investigate in an unbiased systematic study, which poses a challenge 610 not only for achieving statistically meaningful results but also for the findings to be influenced by confounding variables. However, the implementation of orthogonal methods in combination with the independence of the two cohort of patients provide solid evidence of TME heterogeneity at multiple dimensions in addition to reasonable putative mechanisms behind it. Ultimately, we hope that the increasing scientific evidence would lead to better designed clinical trials where 615 TME heterogeneity is further evaluated, data driven combination therapies or novel therapies 
are tested and tissue sections are systematically analyzed and stored for future integrative unbiased analyses.

This study shows that the TME of HGSOC is intrinsically heterogeneous within patients and

620 within tumors, posing an important barrier for the successful application of therapies that target the TME, like checkpoint blockade immunotherapy. By controlling patient dependency and accounting for intra-patient TME heterogeneity, insights into potential mechanisms driving TME heterogeneity were obtained, putting forward new therapeutic strategies to be explored in future studies. Furthermore, the induced immunogenicity upon NACT treatment was only unmasked

625 after taking into account the TME heterogeneity, which otherwise acts as a confounding variable. Despite high rates of response to initial treatment, HGSOC has a high recurrence rate and has yet to show significant response to available immunotherapeutic agents. Exploring new combination therapies and novel therapeutic targets based on a greater understanding of the TME has the potential to change the current paradigm of treatment, and hopefully improve 630 clinical outcomes in this disease. 


\section{METHODS}

\section{Contact for Reagent and Resource Sharing}

Further information and requests for resources and reagents should be directed to and will be fulfilled by the Lead Contact, Martin L. Miller (martin.miller@cruk.cam.ac.uk).

\section{Experimental Model and Subject Details}

\section{Patients}

All patients had stage IIIC or IV high grade serous ovarian cancer as assessed by a pathologist specialized in gynecologic malignancies. Patients signed written consent to Institutional Review Board (IRB)-approved bio-specimen protocols.

\section{Treatment-naive cohort}

For the treatment-naive cohort, 25 patients were consented to the study between August 2014 and March 2016. Out of these patients, 17 were excluded as they either a) withdrew from the study $(n=3) ;$ b) the final pathology was not HGSOC $(n=5) ; c)$ the patients had disease progression upon review of study imaging and underwent neoadjuvant chemotherapy instead of

650 primary cytoreductive surgery $(n=5)$; d) inadequate image-guided tissue sampling due to friable tissue $(n=2)$; e) research imaging studies not performed due to patient cancellation $(n=2)$. The final treatment-naive study population consisted of 8 patients with histopathologically-confirmed diagnosis of HGSOC (Supplementary Table 1A-B). Each patient underwent multi-parametric MRI (mpMRI) of the abdomen and pelvis and 18F-FDG PET/CT within 7 days immediately 655 preceding the primary cytoreductive surgery. Volumetric regions of interest (VOI) were outlined 
on both axial T2-weighted MR images and PET images, covering both the primary and metastatic lesions, using ImageJ (U.S. National Institutes of Health) by a board certified radiologist with special expertise in ovarian cancer imaging. The tumor regions outlined on MRI were co-registered with those outlined on PET.

\section{0}

\section{Neoadjuvant chemotherapy cohort}

For the neoadjuvant chemotherapy cohort a previously established institutional database identified 152 patients with HGSOC who underwent neoadjuvant chemotherapy between 2008 and 2013. Of these patients, 149 went on to interval debulking surgery. 48 of these patients had adequate pre and post treatment formalin fixed paraffin embedded tissue samples available. All pretreatment specimens were obtained either through core biopsy or laparoscopic biopsy, and all post treatment specimens were obtained at the time of laparotomy for interval debulking surgery. Choice of chemotherapy was at the clinician's discretion, but all patients in the cohort received a platinum and taxane based regimen (Supplementary Table 5A). 40 of these paired samples yielded data for analysis, 17 of these pairs were site-matched, meaning that pre-

670 treatment and post-treatment specimens were taken from the same anatomic site, while 23 were site-unmatched. Gene expression and TCRseq data were generated for 28 and 37 pairs respectively (Supplementary Table 5B). Samples with very low TCR sequences ( $n=5$ samples, 10 pairs) were not included in the downstream analyses as the confidence of TCR clonality is low. 


\section{Method Details}

\section{Image acquisition and analysis}

The quantitative diffusion parameters $D$ (diffusion coefficient) and $f$ (the volume fraction of the blood flowing through the microvessels) derived from the intravoxel incoherent motion (IVIM)

680 MRI (Le Bihan et al. 1988) and dynamic contrast-enhanced (DCE) MRI (Tofts 1997) parameter Ktrans (volume transfer constant between the blood plasma and the extravascular extracellular space) were generated voxel-wise using a dedicated in-house software written in Matlab (Mathworks Inc., Natick, MA, USA). The Standardized Uptake Values (SUV) of the voxels contained within each lesion on PET were also calculated (Kinahan et al. 2009). k-means

685 clustering algorithm (Carano et al. 2004) of the $D, f, K^{\text {trans }}$ and SUV voxels, with the number of clusters $(k)$ being fixed to $k=3$ was used to identify imaging clusters/habitats. The mean and standard deviation (mean \pm std. dev.) of these parameters for each cluster were calculated. To establish coherence across patients (i.e. to label each cluster with the $\alpha, \beta, y$ greek letters, such that across patients clusters would have similar imaging features), the intra-cluster distance was

690 calculated for each cluster. The greek letter of the clusters for each patient was assigned based on the relative value of the intra-cluster distance. Specifically, for each patient, $\beta$ was assigned to the cluster which had the highest intra-cluster distance for that patient; $\gamma$ was assigned to the cluster with intermediate intra-cluster distance, and a to the cluster with the lowest intra-cluster distance.

\section{Custom made 3D Tumor Moulds}

For each patient, custom made 3 dimensional (3D) moulds [REF:Weigelt B, Vargas AH, Selenica P, Geyer FC, Mazaheri Y, Blecua P, Conlon N, Hoang LN, Jungbluth AA, Snyder A, Ng CKY, Papanastasiou AD, Sosa RE, Soslow RA, Chi DS, Gardner GJ, Shen R, Reis-Filho 
700 cancer. BJC (under review).] were printed based on manual segmentation of the ovarian mass and metastatic implants on axial T2-weighted MR images. The lesions were outlined on every axial slice and automatically converted into 3D models using open source software (MIPAV, National Institutes for Health, Center for Information Technology). The final 3D models of each lesion were imported into OpenSCAD (OpenSCAD, The OpenSCAD Developers), a 3D CAD

705 modeling software, which was used to create an internal cavity that exactly shaped each lesion according to the MRI shape and contour. The custom-made 3D tumor moulds were printed using a 3D printer (MakerBot Replicator 2, MakerBot, Brooklyn, NY). The slits for slicing each lesion were placed and labelled into the molds at $10 \mathrm{~mm}$ intervals corresponding to the slice thickness and locations of the axial T2W-weighted MR images. The mould was also labeled with

710 left, right, anterior, posterior, superior and inferior markers to allow for proper orientation when collecting samples in the operating room.

\section{Cluster guided specimen sampling}

All 3D moulds containing the specimens were taken to the histopathology department where each lesion was sampled by a pathology fellow. Each tumor was sectioned through the mould 715 and samples were taken according to the imaging habitats/clusters defined above. Half of the sample was sent for histopathology and the other one for immunogenomic analysis.

\section{Immunofluorescent Staining}

The immunofluorescent staining was performed at Molecular Cytology Core Facility of Memorial Sloan Kettering Cancer Center using Discovery XT processor (Ventana Medical Systems). The 720 tissue sections were deparaffinized with EZPrep buffer (Ventana Medical Systems), antigen retrieval was performed with CC1 buffer (Ventana Medical Systems). Sections were blocked for 
30 minutes with Background Buster solution (Innovex), followed by avidin-biotin blocking for 8 minutes (Ventana Medical Systems).

725 Multiplex immunofluorescence stainings were performed as previously described (Yarilin et al. 2015). Slides were incubated with anti-FoxP3 (Abcam, cat\#ab20034, 5 ug/ml) for 4 hours, followed by 60 minutes incubation with biotinylated horse anti-mouse IgG (Vector Labs, cat\# MKB-22258) at 1:200 dilution. The detection was performed with Streptavidin-HRP D (part of DABMap kit, Ventana Medical Systems), followed by incubation with Tyramide Alexa Fluor 488 730 (Invitrogen, cat\# T20922) prepared according to manufacturer instruction with predetermined dilutions. Next, sections were incubated with anti-CD4 (Ventana, cat\#790-4423, 0.5ug/ml) for 5 hours, followed by 60 minutes incubation with biotinylated goat anti- rabbit lgG (Vector, cat \# PK6101) at 1:200 dilution. The detection was performed with Streptavidin-HRP D (part of DABMap kit, Ventana Medical Systems), followed by incubation with Tyramide Alexa 568 735 (Invitrogen, cat\# T20914) prepared according to manufacturer instruction with predetermined dilutions. Finally, sections were incubated with anti-CD8 (Ventana, cat\#790-4460, 0.07ug/ml) for 5 hours, followed by 60 minutes incubation with biotinylated goat anti- rabbit IgG (Vector, cat \# PK6101) at 1:200 dilution. The detection was performed with Streptavidin-HRP D (part of DABMap kit, Ventana Medical Systems), followed by incubation with Tyramide Alexa 647 740 (Invitrogen, cat\# T20936) prepared according to manufacturer instruction with predetermined dilutions. After staining slides were counterstained with DAPI (Sigma Aldrich, cat\# D9542, 5 $\mathrm{ug} / \mathrm{ml}$ ) for $10 \mathrm{~min}$ and coverslipped with Mowiol.

Stained slides were digitized using Pannoramic Flash 250 (3DHistech, Hungary) using $74520 x / 0.8 N A$ objective. Regions of interest were drawn on the scanned images using Pannoramic Viewer (3DHistech, Hungary) and exported into tiff images. ImageJ/FIJI was used to segment DAPI-stained nuclei and count the cells with positive signal. 


\section{Nucleic Acid Isolation and Quantification}

DNA and RNA were extracted from tumor areas delineated as tumor nests on H\&E slides

750 reviewed by a pathologist specialized in gynecologic malignancies using the DNeasy® and RNeasy ${ }^{\circledR}$ (Qiagen) assays, respectively. RNA expression was assessed using the human Affymetrix Clariom D Pico assay (Thermo Fisher Scientific).

\section{T-Cell Receptor Sequencing}

High-throughput in-situ sequencing of the T cell receptors present in the samples and blood of 755 the patient was performed using the immunoSEQ assay platform (Adaptive Biotechnologies).

\section{Quantification and Statistical Analysis}

\section{Gene expression analysis}

RNA expression was assessed using the human Affymetrix Clariom D Pico assay. Arrays were weighted scatterplot smoothing (LOESS) normalization across samples was implemented (Gautier et al. 2004) using:

\# $\mathrm{R} 3.5 .0$ 


\section{Single-sample gene set enrichment analysis}

Single-sample gene set enrichment analysis (Barbie et al. 2009), a modification of standard GSEA (Subramanian et al. 2005), was performed on RNA measurements for each sample using 770 the GSVA package version 1.28.0 (Hänzelmann, Castelo, and Guinney 2013) in R version 3.5.0 with parameters: method $=$ 'ssgsea', and tau $=0.25$. Normalized enrichment scores were generated for the hallmark gene sets (Arthur Liberzon et al. 2015), immune and stromal signatures (Yoshihara et al. 2013), TME cell gene sets obtained from previous publications (Bindea et al. 2013; Davoli et al. 2017), as well as the consensus TME gene sets (Figure S3A).

775 Hallmark gene sets were obtained from MSigDB database version 6.1 (A. Liberzon et al. 2011).

\section{Tumor purity and immune cell gene-expression score}

Tumor purity and total immune component in the tumor samples were analyzed using the ESTIMATE algorithm method version 1.0.13 (Yoshihara et al. 2013) on the gene expression data using the option: platform = 'affymetrix' for the cohort samples and platform = 780 'illumina' for TCGA OV samples, in R version 3.5.0.

\section{Dimensionality reduction}

The t-distributed Stochastic Neighbor Embedding (t-SNE) and principal component analysis dimensionality reduction methods were implemented in python version 3.6 .5 using the sklearn.manifold.TSNE and sklearn.decomposition. PCA functions from the sklearn

785 version 0.19.1 package (Pedregosa et al. 2011). PCA was computed after sample wise standardization. Functions were used as follows:

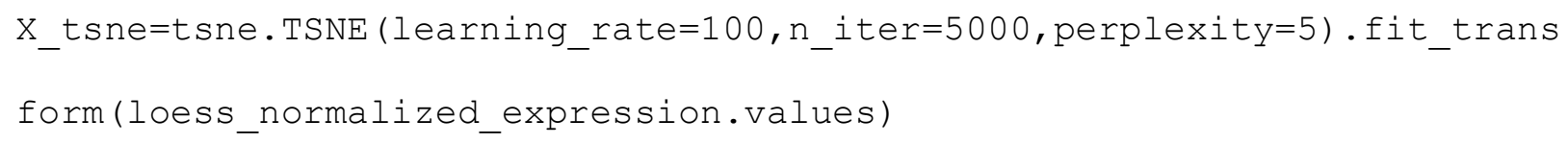


Analysis of T cell infiltration between cases, sites, and habitats

A linear mixed effects model analysis was performed to evaluate if there were significant differences in T cell infiltration subsets between patients, sites within patients, and habitats within tumors, and to assess whether the differences were other than random. Due to data

795 nested dependency we employed a linear mixed effects model under the Ime4 R package (Bates et al. 2015). Cases were considered a random factor, while sites and habitats were considered fixed factors as follows:

\# R 3.5 .0

glmer.fitla <- glmer(cbind(cd8_counts, total_cells - cd8_counts)

site*habitats+(1|case), family = binomial, data = data)

\section{TME cell deconvolution methods}

Cell deconvolution methods were used to estimate levels of non-cancerous cells in the TME.

805 The methods employed were CIBERSORT (Newman et al. 2015), MCP-counter (Becht et al. 2016), TIMER (B. Li et al. 2016), xCELL (Aran, Hu, and Butte 2017), as well as gene sets collected from two previous publications (Bindea et al. 2013; Davoli et al. 2017).

CIBERSORT analysis was performed using the CIBERSORT R script as follows:

\# $\mathrm{R} \quad 3.5 .0$ 
bioRxiv preprint doi: https://doi.org/10.1101/441428; this version posted October 17, 2018. The copyright holder for this preprint (which was not certified by peer review) is the author/funder, who has granted bioRxiv a license to display the preprint in perpetuity. It is made available under aCC-BY-NC-ND 4.0 International license.

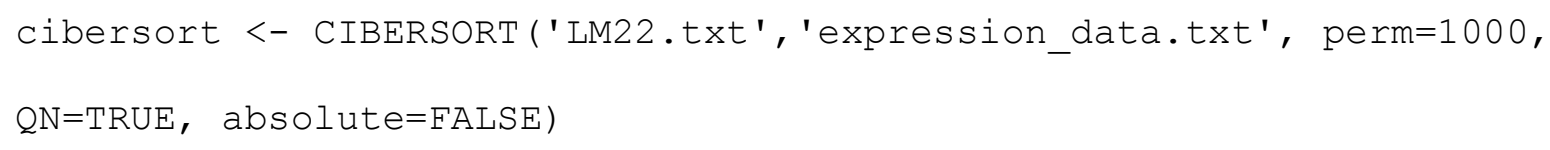

815

MCP-counter analysis was performed as follows:

\# R 3.5 .0

library(MCPCounter) \# version 1.1 .0

exp_data = read.table(expression_data.txt, header $=T, \operatorname{sep}={ }^{\prime} \backslash t^{\prime}$, row. names=' Hugo_Symbol' '

mcp $=$ MCPcounter.estimate (exp_data, featuresType=c ("affy133P2_probesets", "HUGO_symbols", "ENTREZ_ID") [2] , probesets=read.table (curl ("http://raw.githubusercontent.com/ebecht/MCPcounter /master/Signatures/probesets.txt"), sep="\t", stringsAsFactors=FALSE, colClasses ="character"), genes=read.table (curl ("http: //raw.githubusercontent.com/ebecht/MCPcounter/mas ter/Signatures/genes.txt"), sep="\t", stringsAsFactors=FALSE, header=TRUE, colCla sses="character", check . names=FALSE) )

830 The TIMER web server (https://cistrome.shinyapps.io/timer/) was used for deconvolution of TME cells (T. Li et al. 2017).

The xCELL web server version 1.1 (http://xcell.ucsf.edu/) was used for deconvolution of TME cells.

For the Bindea et al. and Davoli et al. gene sets, standard SSGSEA analysis was performed as previously described. 


\section{Consensus TME cell gene sets}

To generate the consensus TME gene sets we identified cell types that were deconvoluted by at

840 least 2 different methods, leading to 18 different cell types. We then intersected the gene sets that the different methods considered for the deconvolution of such cell types. To intersect genes used in CIBERSORT, we first filtered out genes whose weight was below 1.96 standard deviations of the mean for each of CIBERSORT cell types. In addition we combined activated states into the corresponding cell type. The only activated stated included was cytotoxic cells,

845 which would include CD8 and/or NK cells in their activated stated. The intersected genes were used to represent each cell type, and genes with a higher Pearson's correlation coefficient than -0.2 and a $p$-value $\leq 0.05$ with tumor purity as defined by TIMER were filtered out from the gene sets (B. Li et al. 2016). Finally, ssGSEA was employed to calculate NES for each cell type as described above (Figure S3A).

\section{TME cell deconvolution benchmarks}

\section{T cell subsets immunofluorescent staining benchmark}

We correlated the CD8, CD4, Tregs infiltration counts with the deconvolution scores generated by ESTIMATE, CIBERSORT, MCP-counter, Bindea et al., Davoli et al., TIMER, xCELL, and the consensus TME scores. For the immune score comparison, all the genes used for the

855 deconvolution for each method were aggregated together into one single gene set per method except for CIBERSORT. CIBERSORT deconvolution - $\log _{10}(p$-values) were used as a metric for immune score comparison. CD8, CD4, and Treg counts from IF data were summed and used for the comparison. Because the methods have different scoring systems and ranges we standardized (z-score) the scores to be able to compare the results across methods together. 860 For each tumor, multiple IF-stained sections were quantified for TILs, and we correlated all the 
regions quantified with the deconvolution scores of each tumor, explaining the vertical patterns observed in figure 3A. Spearman's rank correlation was performed for each comparison and FDR $p$-value correction was applied.

\section{TCGA OV leukocyte methylation benchmark}

865 As an independent benchmark we used leukocyte methylation scores of TCGA ovarian cancer samples (Cancer Genome Atlas Research Network 2011). TCGA ovarian cancer RNAseq data was retrieved from the cBioPortal (Cerami et al. 2012; Gao et al. 2013) web server version 1.6.2 (http://www.cbioportal.org/).

870 First, deconvolution of cell types was performed using the different methods listed above. Spearman's rank correlations were calculated between CD8 T cell scores and the leukocyte methylation score of TCGA ovarian cancer samples, and FDR p-value correction was applied.

We further fitted multiple linear regression models to each method deconvoluted cell types

875 (Figure S3B). We compared the proportion of leukocyte methylation score variance that is explained by the unsupervised selected immune cells (adjusted R-squared), as well as the relative quality of the models by considering goodness of fit and model simplicity after BoxCox transformation of the leukocyte methylation scores to meet the normality of residuals assumption. As a sensitivity analysis we log transformed the leukocyte methylation scores

880 before performing the linear regression models. In both analyses (BoxCox and log-transform), stepwise Akaike information criterion variable selection was performed once normality and heteroscedasticity assumptions were checked. Akaike information criterion (AIC) and Bayesian information criterion (BIC) were employed independently to compare the fitted models for each method. Both AIC and BIC quantify information loss and penalize the number of variables.

885 Thus, the trade-off between goodness of fit and model simplicity across methods was 
evaluated, allowing us to quantify and minimize information loss. The R version and packages used for this analysis were $\mathrm{R}$ version 3.5.0, gamlss_5.0-8 (Stasinopoulos, Mikis Stasinopoulos, and Rigby 2007), leaps_3.0, car_3.0-0 (Fox and Weisberg 2011), and MASS_7.3-50 (Venables and Ripley 2002).

890 Differential expression analysis

Tumor samples from the treatment-naive cohort were divided into high and low purity classes taking as a cutoff the median of tumor purity calculated for the tumor samples using ESTIMATE (Yoshihara et al. 2013). Then a differential expression analysis was performed using the $R$ packages limma_3.36.1 (Ritchie et al. 2015) and Biobase_2.40.0 (Huber et al.

$8952015)$. Patient dependency was taken into account as follows:

\# $\mathrm{R} 3.5 .0$

library(limma) \# version limma_3.36.1

library(Biobase) \# version Biobase_2.40.0

900

data<-

read.table ('expression_data.txt', sep=' \t', header=T,row.names='Hugo_Symbol' )

eset<-new("Expressionset", exprs=as.matrix(data))

estimate $<-$

read.table ('estimate_purity_scores', sep=' \t', header=T, row. names='NAME' )

purity<-as.data.frame(ifelse(estimate\$TumorPurity > med_purity,

'high_purity', 'low_purity')) 
bioRxiv preprint doi: https://doi.org/10.1101/441428; this version posted October 17, 2018. The copyright holder for this preprint (which was not certified by peer review) is the author/funder, who has granted bioRxiv a license to display the preprint in perpetuity. It is made available under aCC-BY-NC-ND 4.0 International license.

row. names (purity)<-row. names (estimate)

colnames (purity) [1]<- 'purity'

910

patient_data<-

read.table ('patient_data.txt', sep=' \t', header=T, row . names=' NAME' )

clinical<-merge (patient_data, purity, by= 'row. names' )

row. names (clinical)<-row. names (purity)

clinical\$Row . names<-NULL

915

colnames (clinical) [6]<-'purity'

\# merge factors

clinical<-factor (clinicalspurity)

\# Make design matrix

design <- model.matrix ( 0+clinical)

920

colnames(design) <- levels(clinical)

\# estimate correlation between measurements on same subjects

corfit <- duplicateCorrelation(eset, design,block=patient_data\$case)

\# inter-subject correlation is input into the linear model fit

fit $<-$

lmFit (eset, design,block=patient_data\$case, correlation=corfit\$consensus) 
$\mathrm{cm}<-$ makeContrasts (HighPurityvsLowPurity = high_purity-low_purity,

levels=design)

fit2 <- contrasts.fit(fit, cm)

fit2 $<-$ eBayes (fit2)

results <- decideTests (fit2, adjust.method="fdr")

volcanoplot (fit2)

Gene ontology analysis

Gene ontology analysis of significantly up or down-regulated genes was performed using the Gene Ontology Consortium (Ashburner et al. 2000; The Gene Ontology Consortium 2017) web server (http://www.geneontology.org/). P-value FDR corrections were calculated for this analysis.

\section{ssGSEA of differential expression analysis}

Further, $p$-values for each gene were retrieved and multiplied by -1 if the the $\log _{2}$ change was negative. The list of genes with their associated $p$-value was used to calculate hallmark and 940 consensus TME normalized enrichment scores (NES) through ssGSEA. Hallmark gene sets' NES were normalized by taking the exponential function. Consensus TME gene sets' NES approached normality by taking the natural logarithm. Modified z-score was employed to detect outliers in the hallmarks and consensus TME NES independently, as the modified z-score uses the median and the median absolute deviation (MAD) to robustly measure central tendency and 945 dispersion in small data sets (Iglewicz and Hoaglin 1993).

$$
\text { Modified } z-\text { score }=(0.6745 *(y-\text { median })) / \text { MAD }
$$




\section{Paired gene set comparisons}

\section{Volcano plots}

950 For each of the 52 hallmark and 18 consensus TME gene sets paired comparisons before and after NACT were performed. Equality of variance (Bartlett's test) and normality (Shapiro test, Kolmogorov-Smirnov test, and D-Agostino-Pearson's test) assumptions were checked to select the corresponding paired test (Paired T-test, Welch's T-test, or Wilcoxon signed-rank test). The analysis was performed under python 3.6 .5 and scipy 1.1 .0 (http://www.scipy.org/) ecosystem (Oliphant 2007).

Hotelling's $\mathrm{T}^{2}$ distribution test

Multivariate Hotelling's $\mathrm{T}^{2}$ test was performed to compare difference of CD8, NK, and cytotoxic consensus TME gene sets NES between pre- and post-NACT tumors as follows:

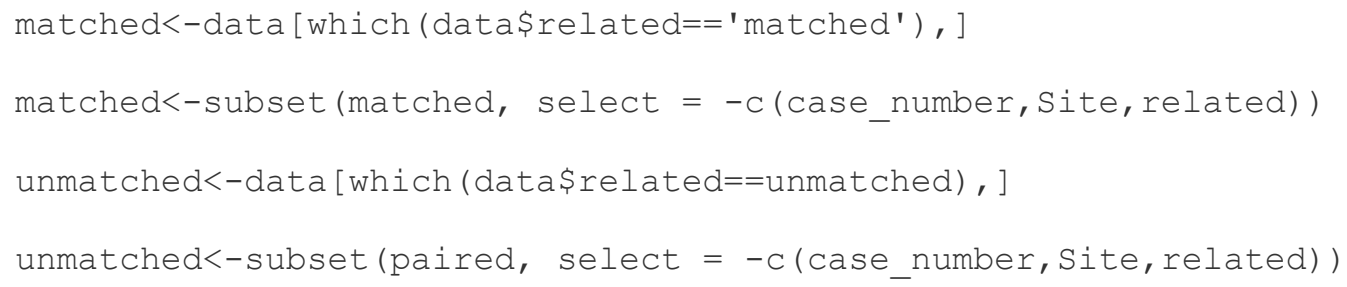

970

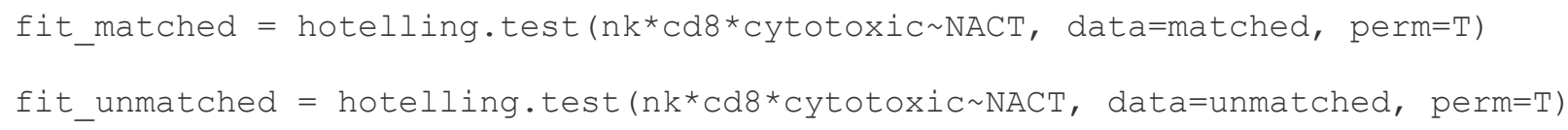


plot (fit_matched)

975 plot(fit_unmatched)

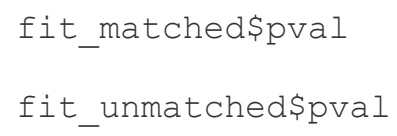

\section{TCR sequencing analysis}

980 Analysis of the sequences was performed on the immunoSEQ ANALYZER 3.0 (Adaptive biotechnologies). T cell counts and TCR clonality were retrieved for statistical comparisons. T cell counts are derived from quantitative immunoSequencing of the TCRB loci, in which the internal controls allow precise quantitation of sequence counts based on reads. Nucleated cell counts are determined by sequencing housekeeping genes. The fraction of $T$ cells is

985 determined by dividing the T cell count by the nucleated cell counts. Values for TCR productive clonality range from 0 to 1 . Values near 1 represent samples with one or a few predominant rearrangements (monoclonal or oligoclonal samples) dominating the observed repertoire. TCR productive clonality values near 0 represent more polyclonal samples. TCR productive clonality is calculated by normalizing productive entropy using the total number of unique productive 990 rearrangements and subtracting the result from 1.

\section{LASSO regression and post-selection inference}

Least absolute shrinkage and selection operator (LASSO) regression analysis was performed using the glmnet R package (Friedman, Hastie, and Tibshirani 2010). Hallmark and consensus TME cell type NES of pre-NACT samples were used independently as explanatory variables, 995 and the $\log _{2}$ of the ratio post/pre NACT TCR clonality as response variable. 
bioRxiv preprint doi: https://doi.org/10.1101/441428; this version posted October 17, 2018. The copyright holder for this preprint (which was not certified by peer review) is the author/funder, who has granted bioRxiv a license to display the preprint in perpetuity. It is made available under aCC-BY-NC-ND 4.0 International license.

\# $\mathrm{R} 3.5 .0$

library(glmnet) \# version glmnet_2.0-16

1000

$\operatorname{dataH}<-$

read.table('Hallmarks_TCRdiff.txt', sep=' \t', header=T, row.names=' ID')

dataC $<-$

read. table('ConsensusTME TCRdiff.txt', sep=' \t', header=T, row. names='ID')

1005

h<-as.matrix (subset (dataH, select $=-c($ clon_dif) $)$ )

$c<-a s . m a t r i x($ subset (datac, select $=-c($ clon_dif))

$y<-a s . m a t r i x\left(d a t a H \$ c l o n \_d i f\right)$

hallmark_fit<-glmnet(h,y,family='gaussian', alpha=1, standardize=T)

1010

tmecells_fit<-glmnet (c,y, family='gaussian', alpha=1, standardize=T)

\# VARIABLE SELECTION

hallmark_cvfit=cv.glmnet (h,y,family="gaussian", type.measure="mse", alpha=1, nfold=10)

1015 tmecells_cvfit=cv.glmnet (c,y,family="gaussian", type.measure="mse",alpha=1,

$\mathrm{nfold}=10$ )

coef(hallmark_cvfit, $s=$ "lambda.min")

coef(tmecells_cvfit, $s=$ "lambda.min")

library ("selectiveInference") \# version selectiveInference_1.2.4 postselinf_h = fixedLassoInf(h,y,beta_hat $[-1]$, lambda,family="gaussian") postselinf_c = fixedLassoInf(c,y,beta_hat[-1], lambda,family="gaussian") 


\section{Data and Software Availability}

Requests for additional data and custom code should be directed to the corresponding authors.

\section{Immunofluorescence staining images}

1030 The immunofluorescence images discussed in this study will be provided upon request to the Lead Contact in the Data and Software Availability section.

\section{Microarray data}

Microarray data is currently under submission to the GEO database.

\section{TCR sequencing data}

1035 The TCR sequencing data discussed in this study will be provided upon request to the Lead Contact in the Data and Software Availability section.

\section{ACKNOWLEDGEMENTS}

We acknowledge Tony Wu for his insightful comments on the manuscript. A.S. was supported by grants from the Marsha Rivkin Organization, Society of Memorial Sloan Kettering,

1040 Translational and Integrative Medicine Research Fund (MSKCC), Kaleidoscope of Hope, and a MSKCC Cancer Center Support Grant of the National Institutes of Health/National Cancer Institute (P30 CA008748). M.L.M. was supported by Cancer Research UK core grant (C14303/A17197). A.J.S. was supported by a doctoral fellowship from the Cancer Research UK Cambridge Institute and the Mexican National Council of Science and Technology (CONACyT). 


\section{CONTRIBUTIONS}

CRediT standard taxonomy

http://dictionary.casrai.org/Contributor Roles

Conceptualization [Ideas; formulation or evolution of overarching research goals and aims] AS, AJS, MLM, ES

Data curation [Management activities to annotate (produce metadata), scrub data and maintain

1055 research data (including software code, where necessary for interpreting the data itself) for initial use and later re-use]

AJS, KL, PC

Formal analysis [Application of statistical, mathematical, computational, or other formal

1060 techniques to analyse or synthesize study data]

AJS

Funding acquisition [Acquisition of the financial support for the project leading to this publication]

Investigation [Conducting a research and investigation process, specifically performing the experiments, or data/evidence collection]

PC, KL, TW, YM, IN, BW, DC, ES 
Methodology [Development or design of methodology; creation of models]

ES

Project administration [Management and coordination responsibility for the research activity

1075 planning and execution]

AS, MLM, ES

Resources [Provision of study materials, reagents, materials, patients, laboratory samples, animals, instrumentation, computing resources, or other analysis tools]

1080 AS, MLM, ES

Software [Programming, software development; designing computer programs, implementation of the computer code and supporting algorithms; testing of existing code components] AJS, ES (the radiologic background)

Supervision [Oversight and leadership responsibility for the research activity planning and execution, including mentorship external to the core team]

AS, MLM, ES

1090 Validation [Verification, whether as a part of the activity or separate, of the overall replication/reproducibility of results/experiments and other research outputs] AJS, DLC

Visualization [Preparation, creation and/or presentation of the published work, specifically 1095 visualization/data presentation]

AJS 
Writing original draft [Preparation, creation and/or presentation of the published work, specifically writing the initial draft]

Writing review \& editing [Preparation, creation and/or presentation of the published work by those from the original research group, specifically critical review, commentary or revision] AJS, MLM, AS, KL, ES, JDB 


\section{REFERENCES}

Ahmed, Ahmed Ashour, Dariush Etemadmoghadam, Jillian Temple, Andy G. Lynch, Mohamed

Riad, Raghwa Sharma, Colin Stewart, et al. 2010. “Driver Mutations in TP53 Are Ubiquitous in High Grade Serous Carcinoma of the Ovary." The Journal of Pathology 221 (1): 49-56.

Aldridge, Bree B., John M. Burke, Douglas A. Lauffenburger, and Peter K. Sorger. 2006.

"Physicochemical Modelling of Cell Signaling Pathways." Nature Cell Biology 8 (11): 11951203.

Amé, J. C., V. Rolli, V. Schreiber, C. Niedergang, F. Apiou, P. Decker, S. Muller, T. Höger, J. Ménissier-de Murcia, and G. de Murcia. 1999. "PARP-2, A Novel Mammalian DNA Damage-Dependent poly(ADP-Ribose) Polymerase." The Journal of Biological Chemistry 274 (25): 17860-68.

Aran, Dvir, Zicheng Hu, and Atul J. Butte. 2017. “xCell: Digitally Portraying the Tissue Cellular Heterogeneity Landscape." Genome Biology 18 (1): 220.

1125 Ashburner, M., C. A. Ball, J. A. Blake, D. Botstein, H. Butler, J. M. Cherry, A. P. Davis, et al. 2000. "Gene Ontology: Tool for the Unification of Biology. The Gene Ontology Consortium." Nature Genetics 25 (1): 25-29.

Barbie, David A., Pablo Tamayo, Jesse S. Boehm, So Young Kim, Susan E. Moody, Ian F. Dunn, Anna C. Schinzel, et al. 2009. "Systematic RNA Interference Reveals That Oncogenic KRAS-Driven Cancers Require TBK1.” Nature 462 (7269): 108-12.

Bates, Douglas, Martin Mächler, Ben Bolker, and Steve Walker. 2015. "Fitting Linear MixedEffects Models Usinglme4.” Journal of Statistical Software 67 (1). https://doi.org/10.18637/jss.v067.i01.

Becht, Etienne, Nicolas A. Giraldo, Laetitia Lacroix, Bénédicte Buttard, Nabila Elarouci, Florent Petitprez, Janick Selves, et al. 2016. "Estimating the Population Abundance of Tissue- 
Infiltrating Immune and Stromal Cell Populations Using Gene Expression." Genome Biology $17(1): 218$.

Bindea, Gabriela, Bernhard Mlecnik, Marie Tosolini, Amos Kirilovsky, Maximilian Waldner, Anna C. Obenauf, Helen Angell, et al. 2013. "Spatiotemporal Dynamics of Intratumoral Immune Cells Reveal the Immune Landscape in Human Cancer." Immunity 39 (4): 782-95.

Böhm, Steffen, Anne Montfort, Oliver M. T. Pearce, Joanne Topping, Probir Chakravarty, Gemma L. A. Everitt, Andrew Clear, et al. 2016. "Neoadjuvant Chemotherapy Modulates the Immune Microenvironment in Metastases of Tubo-Ovarian High-Grade Serous Carcinoma." Clinical Cancer Research: An Official Journal of the American Association for $1145 \quad$ Cancer Research 22 (12): 3025-36.

Bowtell, David D., Steffen Böhm, Ahmed A. Ahmed, Paul-Joseph Aspuria, Robert C. Bast Jr, Valerie Beral, Jonathan S. Berek, et al. 2015. "Rethinking Ovarian Cancer II: Reducing Mortality from High-Grade Serous Ovarian Cancer." Nature Reviews. Cancer 15 (11): 66879.

Diagnosis versus Mechanism." Nature Reviews. Immunology 18 (5): 289-90.

Campbell, Peter J., Shinichi Yachida, Laura J. Mudie, Philip J. Stephens, Erin D. Pleasance, Lucy A. Stebbings, Laura A. Morsberger, et al. 2010. “The Patterns and Dynamics of Genomic Instability in Metastatic Pancreatic Cancer." Nature 467 (7319): 1109-13.

1155 Cancer Genome Atlas Research Network. 2011. “Integrated Genomic Analyses of Ovarian Carcinoma." Nature 474 (7353): 609-15.

Cantor, S. B., T. Urano, and L. A. Feig. 1995. "Identification and Characterization of Ral-Binding Protein 1, a Potential Downstream Target of Ral GTPases." Molecular and Cellular Biology 15 (8): 4578-84.

1160 Carano, Richard A. D., Adrienne L. Ross, Jed Ross, Simon P. Williams, Hartmut Koeppen, Ralph H. Schwall, and Nicholas Van Bruggen. 2004. "Quantification of Tumor Tissue 
Populations by Multispectral Analysis." Magnetic Resonance in Medicine: Official Journal of the Society of Magnetic Resonance in Medicine / Society of Magnetic Resonance in Medicine 51 (3): 542-51.

1165 Carter, Scott L., Kristian Cibulskis, Elena Helman, Aaron McKenna, Hui Shen, Travis Zack, Peter W. Laird, et al. 2012. "Absolute Quantification of Somatic DNA Alterations in Human Cancer." Nature Biotechnology 30 (5): 413-21.

Cerami, Ethan, Jianjiong Gao, Ugur Dogrusoz, Benjamin E. Gross, Selcuk Onur Sumer, Bülent Arman Aksoy, Anders Jacobsen, et al. 2012. "The cBio Cancer Genomics Portal: An Open $1170 \quad$ Platform for Exploring Multidimensional Cancer Genomics Data." Cancer Discovery 2 (5): 401-4.

Colak, Selcuk, and Peter ten Dijke. 2017. "Targeting TGF- $\beta$ Signaling in Cancer." Trends in Cancer Research 3 (1): 56-71.

Damsky, William E., David P. Curley, Manjula Santhanakrishnan, Lara E. Rosenbaum, James

1175 T. Platt, Bonnie E. Gould Rothberg, Makoto M. Taketo, et al. 2011. " $\beta$-Catenin Signaling Controls Metastasis in Braf-Activated Pten-Deficient Melanomas." Cancer Cell 20 (6): 74154.

Davies, Stefan M. K., Oliver Rackham, Anne-Marie J. Shearwood, Kristina L. Hamilton, Reena Narsai, James Whelan, and Aleksandra Filipovska. 2009. "Pentatricopeptide Repeat Domain Protein 3 Associates with the Mitochondrial Small Ribosomal Subunit and Regulates Translation." FEBS Letters 583 (12): 1853-58.

Davoli, Teresa, Hajime Uno, Eric C. Wooten, and Stephen J. Elledge. 2017. “Tumor Aneuploidy Correlates with Markers of Immune Evasion and with Reduced Response to Immunotherapy." Science 355 (6322). https://doi.org/10.1126/science.aaf8399.

1185 Fang, Xianjun, Michel Schummer, Muling Mao, Shuangxing Yu, Fazal Haq Tabassam, Ramona Swaby, Yutaka Hasegawa, et al. 2002. "Lysophosphatidic Acid Is a Bioactive Mediator in Ovarian Cancer." Biochimica et Biophysica Acta 1582 (1-3): 257-64. 
Finotello, Francesca, and Zlatko Trajanoski. 2018a. "Quantifying Tumor-Infiltrating Immune Cells from Transcriptomics Data." Cancer Immunology, Immunotherapy: CII, March. https://doi.org/10.1007/s00262-018-2150-z.

Fox, John, and Sanford Weisberg. 2011. An R Companion to Applied Regression. SAGE Publications.

Friedman, Jerome, Trevor Hastie, and Rob Tibshirani. 2010. "Regularization Paths for Generalized Linear Models via Coordinate Descent." Journal of Statistical Software 33 (1): $1-22$.

Frye, Björn C., Sarah Halfter, Sonja Djudjaj, Philipp Muehlenberg, Susanne Weber, Ute Raffetseder, Abdelaziz En-Nia, et al. 2009. "Y-Box Protein-1 Is Actively Secreted through a Non-Classical Pathway and Acts as an Extracellular Mitogen." EMBO Reports 10 (7): 78389.

1200 Gao, Jianjiong, Bülent Arman Aksoy, Ugur Dogrusoz, Gideon Dresdner, Benjamin Gross, S. Onur Sumer, Yichao Sun, et al. 2013. “Integrative Analysis of Complex Cancer Genomics and Clinical Profiles Using the cBioPortal." Science Signaling 6 (269): I1.

Gautier, Laurent, Leslie Cope, Benjamin M. Bolstad, and Rafael A. Irizarry. 2004. "Affy--Analysis of Affymetrix GeneChip Data at the Probe Level." Bioinformatics 20 (3): 307-15.

1205 Gerlinger, Marco, Andrew J. Rowan, Stuart Horswell, M. Math, James Larkin, David Endesfelder, Eva Gronroos, et al. 2012. "Intratumor Heterogeneity and Branched Evolution Revealed by Multiregion Sequencing." The New England Journal of Medicine 366 (10): 883-92.

Geva-Zatorsky, Naama, Erez Dekel, Ariel A. Cohen, Tamar Danon, Lydia Cohen, and Uri Alon. 2010. "Protein Dynamics in Drug Combinations: A Linear Superposition of Individual-Drug Responses." Cell 140 (5): 643-51.

Gounari, Fotini, Sabina Signoretti, Roderick Bronson, Ludger Klein, William R. Sellers, Jennifer Kum, Anja Siermann, Makoto M. Taketo, Harald von Boehmer, and Khashayarsha Khazaie. 
2002. "Stabilization of $\beta$-Catenin Induces Lesions Reminiscent of Prostatic Intraepithelial Neoplasia, but Terminal Squamous Transdifferentiation of Other Secretory Epithelia."

Oncogene 21 (26): 4099-4107.

Gunderson, Camille C., and Kathleen N. Moore. 2015. "Olaparib: An Oral PARP-1 and PARP-2 Inhibitor with Promising Activity in Ovarian Cancer." Future Oncology 11 (5): 747-57.

Hansen, Jean M., Robert L. Coleman, and Anil K. Sood. 2016. "Targeting the Tumour Microenvironment in Ovarian Cancer." European Journal of Cancer 56: 131-43.

Hänzelmann, Sonja, Robert Castelo, and Justin Guinney. 2013. "GSVA: Gene Set Variation Analysis for Microarray and RNA-Seq Data." BMC Bioinformatics 14 (January): 7.

Heng, Tracy S. P., Michio W. Painter, and Immunological Genome Project Consortium. 2008.

“The Immunological Genome Project: Networks of Gene Expression in Immune Cells.”

$1225 \quad$ Nature Immunology 9 (10): 1091-94.

Hirata, Eishu, Maria Romina Girotti, Amaya Viros, Steven Hooper, Bradley Spencer-Dene, Michiyuki Matsuda, James Larkin, Richard Marais, and Erik Sahai. 2015. "Intravital Imaging Reveals How BRAF Inhibition Generates Drug-Tolerant Microenvironments with High Integrin $\beta 1 / F A K$ Signaling." Cancer Cell 27 (4): 574-88.

1230 Hiroyama, M., and T. Takenawa. 1999. "Isolation of a cDNA Encoding Human Lysophosphatidic Acid Phosphatase That Is Involved in the Regulation of Mitochondrial Lipid Biosynthesis." The Journal of Biological Chemistry 274 (41): 29172-80.

Horiba, Naoshi, Satohiro Masuda, Ayako Takeuchi, Daisuke Takeuchi, Masahiro Okuda, and Ken-Ichi Inui. 2003. "Cloning and Characterization of a Novel Na+-Dependent Glucose Transporter (NaGLT1) in Rat Kidney." The Journal of Biological Chemistry 278 (17): 14669-76.

Huber, Wolfgang, Vincent J. Carey, Robert Gentleman, Simon Anders, Marc Carlson, Benilton S. Carvalho, Hector Corrada Bravo, et al. 2015. "Orchestrating High-Throughput Genomic Analysis with Bioconductor." Nature Methods 12 (2): 115-21. 
$1240 \mathrm{Hu}$, Yanzhong, and Nahid F. Mivechi. 2003. "HSF-1 Interacts with Ral-Binding Protein 1 in a Stress-Responsive, Multiprotein Complex with HSP90in Vivo." The Journal of Biological Chemistry 278 (19): 17299-306.

lannello, Alexandre, Thornton W. Thompson, Michele Ardolino, Scott W. Lowe, and David H.

Raulet. 2013. "p53-Dependent Chemokine Production by Senescent Tumor Cells Supports

NKG2D-Dependent Tumor Elimination by Natural Killer Cells." The Journal of Experimental

Medicine 210 (10): 2057-69.

Iglewicz, Boris, and David Caster Hoaglin. 1993. How to Detect and Handle Outliers. Asq Press.

Janssen, Louise M. E., Emma E. Ramsay, Craig D. Logsdon, and Willem W. Overwijk. 2017.

"The Immune System in Cancer Metastasis: Friend or Foe?” Journal for ImmunoTherapy of

Cancer 5 (1). https://doi.org/10.1186/s40425-017-0283-9.

Jiménez-Sánchez, Alejandro, Danish Memon, Stephane Pourpe, Harini Veeraraghavan,

Yanyun Li, Hebert Alberto Vargas, Michael B. Gill, et al. 2017a. "Heterogeneous Tumor-

Immune Microenvironments among Differentially Growing Metastases in an Ovarian

Cancer Patient." Cell 170 (5): 927-38.e20.

1255 Johnson, Brett E., Tali Mazor, Chibo Hong, Michael Barnes, Koki Aihara, Cory Y. McLean,

Shaun D. Fouse, et al. 2014. "Mutational Analysis Reveals the Origin and Therapy-Driven

Evolution of Recurrent Glioma." Science 343 (6167): 189-93.

Kinahan, Paul E., Robert K. Doot, Michelle Wanner-Roybal, Luc M. Bidaut, Samuel G. Armato,

Charles R. Meyer, and Geoffrey McLennan. 2009. "PET/CT Assessment of Response to

Therapy: Tumor Change Measurement, Truth Data, and Error.” Translational Oncology 2

(4): 223-30.

Kirsch, Ilan, Marissa Vignali, and Harlan Robins. 2015. "T-Cell Receptor Profiling in Cancer."

Molecular Oncology 9 (10): 2063-70.

Kitamura, Takanori, Bin-Zhi Qian, and Jeffrey W. Pollard. 2015. “Immune Cell Promotion of 
Kortlever, Roderik M., Nicole M. Sodir, Catherine H. Wilson, Deborah L. Burkhart, Luca

Pellegrinet, Lamorna Brown Swigart, Trevor D. Littlewood, and Gerard I. Evan. 2017. “Myc

Cooperates with Ras by Programming Inflammation and Immune Suppression." Cell 171

(6): 1301-15.e14.

1270 Landgren, Henrik, and Peter Carlsson. 2004. "FoxJ3, a Novel Mammalian Forkhead Gene

Expressed in Neuroectoderm, Neural Crest, and Myotome." Developmental Dynamics: An

Official Publication of the American Association of Anatomists 231 (2): 396-401.

Le Bihan, D., E. Breton, D. Lallemand, M. L. Aubin, J. Vignaud, and M. Laval-Jeantet. 1988.

"Separation of Diffusion and Perfusion in Intravoxel Incoherent Motion MR Imaging."

$1275 \quad$ Radiology 168 (2): 497-505.

Liberzon, Arthur, Chet Birger, Helga Thorvaldsdóttir, Mahmoud Ghandi, Jill P. Mesirov, and

Pablo Tamayo. 2015. "The Molecular Signatures Database Hallmark Gene Set Collection.”

Cell Systems 1 (6): 417-25.

Liberzon, A., A. Subramanian, R. Pinchback, H. Thorvaldsdottir, P. Tamayo, and J. P. Mesirov.

2011. "Molecular Signatures Database (MSigDB) 3.0." Bioinformatics 27 (12): 1739-40.

Li, Bo, Jun S. Liu, and X. Shirley Liu. 2017. "Revisit Linear Regression-Based Deconvolution

Methods for Tumor Gene Expression Data." Genome Biology 18 (1): 127.

Li, Bo, Eric Severson, Jean-Christophe Pignon, Haoquan Zhao, Taiwen Li, Jesse Novak, Peng Jiang, et al. 2016. "Comprehensive Analyses of Tumor Immunity: Implications for Cancer Immunotherapy." Genome Biology 17 (1): 174.

Li, Taiwen, Jingyu Fan, Binbin Wang, Nicole Traugh, Qianming Chen, Jun S. Liu, Bo Li, and X. Shirley Liu. 2017. "TIMER: A Web Server for Comprehensive Analysis of Tumor-Infiltrating Immune Cells." Cancer Research 77 (21): e108-10.

Mariathasan, Sanjeev, Shannon J. Turley, Dorothee Nickles, Alessandra Castiglioni, Kobe Yuen, Yulei Wang, Edward E. Kadel III, et al. 2018. "TGF $\beta$ Attenuates Tumour Response to PD-L1 Blockade by Contributing to Exclusion of T Cells." Nature 554 (7693): 544-48. 
McPherson, Andrew, Andrew Roth, Emma Laks, Tehmina Masud, Ali Bashashati, Allen W. Zhang, Gavin Ha, et al. 2016. "Divergent Modes of Clonal Spread and Intraperitoneal Mixing in High-Grade Serous Ovarian Cancer." Nature Genetics 48 (7): 758-67.

Molinelli, Evan J., Anil Korkut, Weiqing Wang, Martin L. Miller, Nicholas P. Gauthier, Xiaohong Jing, Poorvi Kaushik, et al. 2013. "Perturbation Biology: Inferring Signaling Networks in Cellular Systems." PLoS Computational Biology 9 (12): e1003290.

Muñoz, Ivan M., Thomas MacArtney, Luis Sanchez-Pulido, Chris P. Ponting, Sonia Rocha, and John Rouse. 2012. "Family with Sequence Similarity 60A (FAM60A) Protein Is a Cell CycleFluctuating Regulator of the SIN3-HDAC1 Histone Deacetylase Complex." The Journal of Biological Chemistry 287 (39): 32346-53.

Natrajan, Rachael, Heba Sailem, Faraz K. Mardakheh, Mar Arias Garcia, Christopher J. Tape, Mitch Dowsett, Chris Bakal, and Yinyin Yuan. 2016. “Microenvironmental Heterogeneity Parallels Breast Cancer Progression: A Histology-Genomic Integration Analysis." PLoS Medicine 13 (2): e1001961.

Newman, Aaron M., Andrew J. Gentles, Chih Long Liu, Maximilian Diehn, and Ash A. Alizadeh. 2017. “Data Normalization Considerations for Digital Tumor Dissection." Genome Biology 18 (1): 128.

Newman, Aaron M., Chih Long Liu, Michael R. Green, Andrew J. Gentles, Weiguo Feng, Yue Xu, Chuong D. Hoang, Maximilian Diehn, and Ash A. Alizadeh. 2015. "Robust Enumeration of Cell Subsets from Tissue Expression Profiles." Nature Methods 12 (5): 453-57.

Oliphant, Travis E. 2007. "Python for Scientific Computing." Computing in Science \& Engineering 9 (3): 10-20.

Ovarian Tumor Tissue Analysis (OTTA) Consortium, Ellen L. Goode, Matthew S. Block, Kimberly R. Kalli, Robert A. Vierkant, Wenqian Chen, Zachary C. Fogarty, et al. 2017. "Dose-Response Association of CD8+ Tumor-Infiltrating Lymphocytes and Survival Time in High-Grade Serous Ovarian Cancer.” JAMA Oncology 3 (12): e173290. 
Patch, Ann-Marie, Elizabeth L. Christie, Dariush Etemadmoghadam, Dale W. Garsed, Joshy George, Sian Fereday, Katia Nones, et al. 2015. "Whole-Genome Characterization of Chemoresistant Ovarian Cancer." Nature 521 (7553): 489-94.

Patel, Shetal A., and Andy J. Minn. 2018. "Combination Cancer Therapy with Immune Checkpoint Blockade: Mechanisms and Strategies." Immunity 48 (3): 417-33.

Pielou, E. C. 1966. "Species-Diversity and Pattern-Diversity in the Study of Ecological Succession." Journal of Theoretical Biology 10 (2): 370-83.

1325 Pedregosa, Fabian, Varoquaux, Gael, Gramfort, Alexandre, Michel, Vincent, Thirion, Bertrand, Grisel, Olivier, Mathieu, Blondel, Prettenhofer, Peter, Weiss, Ron, Dubourg, Vincent, Vanderplas, Jake, Passos, Alexandre, Cournapeau, David, Brucher, Matthieu, and Duchesnay, Eduoard. 2011. "Scikit-learn: Machine Learning in Python.” Journal of Machine Learning Research 12: 2825-2830.

1330 Reuben, Alexandre, Christine N. Spencer, Peter A. Prieto, Vancheswaran Gopalakrishnan, Sangeetha M. Reddy, John P. Miller, Xizeng Mao, et al. 2017. “Genomic and Immune Heterogeneity Are Associated with Differential Responses to Therapy in Melanoma." NPJ Genomic Medicine 2 (April). https://doi.org/10.1038/s41525-017-0013-8.

Ritchie, Matthew E., Belinda Phipson, Di Wu, Yifang Hu, Charity W. Law, Wei Shi, and Gordon K. Smyth. 2015. "Limma Powers Differential Expression Analyses for RNA-Sequencing and Microarray Studies." Nucleic Acids Research 43 (7): e47.

Robinson, Dan R., Yi-Mi Wu, Robert J. Lonigro, Pankaj Vats, Erin Cobain, Jessica Everett, Xuhong Cao, et al. 2017. "Integrative Clinical Genomics of Metastatic Cancer.” Nature 548 (7667): 297-303.

1340 Rüegsegger, Ursula, Diana Blank, and Walter Keller. 1998. "Human Pre-mRNA Cleavage Factor Im Is Related to Spliceosomal SR Proteins and Can Be Reconstituted In Vitro from Recombinant Subunits." Molecular Cell 1 (2): 243-53.

Shah, Sohrab P., Ryan D. Morin, Jaswinder Khattra, Leah Prentice, Trevor Pugh, Angela 
Burleigh, Allen Delaney, et al. 2009. "Mutational Evolution in a Lobular Breast Tumour Profiled at Single Nucleotide Resolution." Nature 461 (7265): 809-13.

Sharma, Padmanee, Siwen Hu-Lieskovan, Jennifer A. Wargo, and Antoni Ribas. 2017.

"Primary, Adaptive, and Acquired Resistance to Cancer Immunotherapy." Cell 168 (4):

707-23.

Smith, Karen T., Mihaela E. Sardiu, Skylar A. Martin-Brown, Chris Seidel, Arcady Mushegian, Rhonda Egidy, Laurence Florens, Michael P. Washburn, and Jerry L. Workman. 2012. "Human Family with Sequence Similarity 60 Member A (FAM60A) Protein: A New Subunit of the Sin3 Deacetylase Complex." Molecular \& Cellular Proteomics: MCP 11 (12): 181528.

Spranger, Stefani, Riyue Bao, and Thomas Gajewski. 2014. "Melanoma-Intrinsic $\beta$-Catenin Signaling Prevents T Cell Infiltration and Anti-Tumor Immunity." Journal for ImmunoTherapy of Cancer 2 (Suppl 3): O15.

Spranger, Stefani, Riyue Bao, and Thomas F. Gajewski. 2015. “Melanoma-Intrinsic $\beta$-Catenin Signaling Prevents Anti-Tumour Immunity." Nature 523 (7559): 231-35.

Spranger, Stefani, Daisy Dai, Brendan Horton, and Thomas F. Gajewski. 2017. “TumorResiding Batf3 Dendritic Cells Are Required for Effector T Cell Trafficking and Adoptive T Cell Therapy." Cancer Cell 31 (5): 711-23.e4.

Spranger, Stefani, and Thomas F. Gajewski. 2018. "Impact of Oncogenic Pathways on Evasion of Antitumour Immune Responses." Nature Reviews. Cancer, January. https://doi.org/10.1038/nrc.2017.117.

1365 Spranger, Stefani, Jason J. Luke, Riyue Bao, Yuanyuan Zha, Kyle M. Hernandez, Yan Li, Alexander P. Gajewski, Jorge Andrade, and Thomas F. Gajewski. 2016. "Density of Immunogenic Antigens Does Not Explain the Presence or Absence of the T-Cell-inflamed Tumor Microenvironment in Melanoma." Proceedings of the National Academy of Sciences 113 (48): E7759-68. 
1370 Sridharan, Vishwajith, Evisa Gjini, Xiaoyun Liao, Nicole G. Chau, Robert I. Haddad, Mariano Severgnini, Peter Hammerman, et al. 2016. "Immune Profiling of Adenoid Cystic Carcinoma: PD-L2 Expression and Associations with Tumor-Infiltrating Lymphocytes." Cancer Immunology Research 4 (8): 679-87.

Stasinopoulos, D. Mikis, D. Mikis Stasinopoulos, and Robert A. Rigby. 2007. "Generalized Additive Models for Location Scale and Shape (GAMLSS) inR." Journal of Statistical Software 23 (7). https://doi.org/10.18637/jss.v023.i07.

Subramanian, Aravind, Pablo Tamayo, Vamsi K. Mootha, Sayan Mukherjee, Benjamin L. Ebert, Michael A. Gillette, Amanda Paulovich, et al. 2005. "Gene Set Enrichment Analysis: A Knowledge-Based Approach for Interpreting Genome-Wide Expression Profiles." Proceedings of the National Academy of Sciences of the United States of America 102 (43): 15545-50.

Tauriello, Daniele V. F., Sergio Palomo-Ponce, Diana Stork, Antonio Berenguer-Llergo, Jordi Badia-Ramentol, Mar Iglesias, Marta Sevillano, et al. 2018. "TGF $\beta$ Drives Immune Evasion in Genetically Reconstituted Colon Cancer Metastasis." Nature 554 (7693): 538-43.

1385 The Gene Ontology Consortium. 2017. “Expansion of the Gene Ontology Knowledgebase and Resources." Nucleic Acids Research 45 (D1): D331-38.

Tofts, P. S. 1997. “Modeling Tracer Kinetics in Dynamic Gd-DTPA MR Imaging.” Journal of Magnetic Resonance Imaging: JMRI 7 (1): 91-101.

Tu, J. C., B. Xiao, J. P. Yuan, A. A. Lanahan, K. Leoffert, M. Li, D. J. Linden, and P. F. Worley. 1998. "Homer Binds a Novel Proline-Rich Motif and Links Group 1 Metabotropic Glutamate Receptors with IP3 Receptors." Neuron 21 (4): 717-26.

Venables, W. N., and B. D. Ripley. 2002. Modern Applied Statistics with S. Springer. Wang, Weimin, llona Kryczek, Lubomír Dostál, Heng Lin, Lijun Tan, Lili Zhao, Fujia Lu, et al. 2016. "Effector T Cells Abrogate Stroma-Mediated Chemoresistance in Ovarian Cancer." $1395 \quad$ Cell $165(5):$ 1092-1105. 
Weidensdorfer, Doreen, Nadine Stöhr, Anne Baude, Marcell Lederer, Marcel Köhn, Angelika Schierhorn, Sabine Buchmeier, Elmar Wahle, and Stefan Hüttelmaier. 2009. "Control of cMyc mRNA Stability by IGF2BP1-Associated Cytoplasmic RNPs." RNA 15 (1): 104-15.

Xiao, B., J. C. Tu, R. S. Petralia, J. P. Yuan, A. Doan, C. D. Breder, A. Ruggiero, A. A. Lanahan, R. J. Wenthold, and P. F. Worley. 1998. "Homer Regulates the Association of Group 1 Metabotropic Glutamate Receptors with Multivalent Complexes of Homer-Related, Synaptic Proteins." Neuron 21 (4): 707-16.

Xue, Wen, Lars Zender, Cornelius Miething, Ross A. Dickins, Eva Hernando, Valery Krizhanovsky, Carlos Cordon-Cardo, and Scott W. Lowe. 2007. “Senescence and Tumour 1405 Clearance Is Triggered by p53 Restoration in Murine Liver Carcinomas." Nature 445 (7128): 656-60.

Yarilin, Dmitry, Ke Xu, Mesruh Turkekul, Ning Fan, Yevgeniy Romin, Sho Fijisawa, Afsar Barlas, and Katia Manova-Todorova. 2015. "Machine-Based Method for Multiplex in Situ Molecular Characterization of Tissues by Immunofluorescence Detection." Scientific Reports 5 $1410 \quad$ (March): 9534.

Yates, Lucy R., Moritz Gerstung, Stian Knappskog, Christine Desmedt, Gunes Gundem, Peter Van Loo, Turid Aas, et al. 2015. "Subclonal Diversification of Primary Breast Cancer Revealed by Multiregion Sequencing." Nature Medicine 21 (7): 751-59.

Yoshihara, Kosuke, Maria Shahmoradgoli, Emmanuel Martínez, Rahulsimham Vegesna, Hoon Kim, Wandaliz Torres-Garcia, Victor Treviño, et al. 2013. “Inferring Tumour Purity and Stromal and Immune Cell Admixture from Expression Data." Nature Communications 4 (October). Nature Publishing Group: 2612.

Zhang, Allen W., Andrew McPherson, Katy Milne, David R. Kroeger, Phineas T. Hamilton, Alex Miranda, Tyler Funnell, et al. 2018. "Interfaces of Malignant and Immunologic Clonal Dynamics in Ovarian Cancer." Cell, May. https://doi.org/10.1016/j.cell.2018.03.073. Zhang, Lin, Jose R. Conejo-Garcia, Dionyssios Katsaros, Phyllis A. Gimotty, Marco Massobrio, 
bioRxiv preprint doi: https://doi.org/10.1101/441428; this version posted October 17, 2018. The copyright holder for this preprint (which was not certified by peer review) is the author/funder, who has granted bioRxiv a license to display the preprint in perpetuity. It is made available under aCC-BY-NC-ND 4.0 International license.

Giorgia Regnani, Antonis Makrigiannakis, et al. 2003. "Intratumoral T Cells, Recurrence, and Survival in Epithelial Ovarian Cancer." The New England Journal of Medicine 348 (3): 203-13.

1425 Zheng, Siyuan. 2017a. "Benchmarking: Contexts and Details Matter." Genome Biology 18 (1): 129. 